\title{
Cosmological Constraints from Sunyaev- Zel'dovich-selected Clusters with X-Ray Observations in the First 178 deg2 of the South Pole Telescope Survey
}

\section{Citation}

Benson, B. A., T. de Haan, J. P. Dudley, C. L. Reichardt, K. A. Aird, K. Andersson, R. Armstrong, et al. 2013. "Cosmological Constraints from Sunyaev-Zel'dovich-selected Clusters with X-Ray Observations in the First 178 deg2 of the South Pole Telescope Survey." The Astrophysical Journal 763 (2) (February 1): 147. doi:10.1088/0004-637x/763/2/147. http:// dx.doi.org/10.1088/0004-637X/763/2/147.

\section{Published Version}

doi:10.1088/0004-637x/763/2/147

\section{Permanent link}

http://nrs.harvard.edu/urn-3:HUL.InstRepos:11857775

\section{Terms of Use}

This article was downloaded from Harvard University's DASH repository, and is made available under the terms and conditions applicable to Other Posted Material, as set forth at http:// nrs.harvard.edu/urn-3:HUL.InstRepos:dash.current.terms-of-use\#LAA

\section{Share Your Story}

The Harvard community has made this article openly available.

Please share how this access benefits you. Submit a story.

\section{Accessibility}




\title{
COSMOLOGICAL CONSTRAINTS FROM SUNYAEV-ZEL'DOVICH-SELECTED CLUSTERS WITH X-RAY OBSERVATIONS IN THE FIRST $178 \mathrm{deg}^{2}$ OF THE SOUTH POLE TELESCOPE SURVEY
}
B. A. Benson ${ }^{1,2}$, T. de HaAn ${ }^{3}$, J. P. Dudley ${ }^{3}$, C. L. Reichardt ${ }^{4}$, K. A. Aird ${ }^{5}$, K. Andersson An, $^{6}$, R. Armstrong $^{8}$, M. L. N. AshbY ${ }^{9}$, M. Bautz ${ }^{7}$, M. BAyliss ${ }^{10}$, G. Bazin ${ }^{6,11}$, L. E. BleEM ${ }^{1,12}$, M. Brodwin ${ }^{13}$, J. E. CARLSTrom ${ }^{1,2,12,14,15}$, C. L. Chang $^{1,2,15}$, H. M. Cho ${ }^{16}$, A. Clocchiatti ${ }^{17}$, T. M. Crawford ${ }^{1,14}$, A. T. Crites ${ }^{1,14}$, S. Desai ${ }^{6,11}$, M. A. Dobbs $^{3}$, R. J. Foley ${ }^{9}$, W. R. Forman ${ }^{9}$, E. M. George ${ }^{4}$, M. D. Gladders ${ }^{1}{ }^{14}$, A. H. Gonzalez ${ }^{18}$, N. W. Halverson ${ }^{19}$, N. Harrington ${ }^{4}$, F. W. High ${ }^{1,14}$, G. P. Holder ${ }^{3}$, W. L. Holzapfel ${ }^{4}$, S. Hoover ${ }^{1,2}$, J. D. Hrubes ${ }^{5}$, C. Jones ${ }^{9}$, M. JoY ${ }^{20}$, R. Keisler ${ }^{1,12}$, L. KNOX ${ }^{21}$,

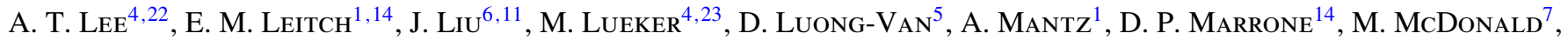
J. J. McMahon ${ }^{1,2,25}$, J. Mehl ${ }^{1,14}$, S. S. Meyer ${ }^{1,2,12,14}$, L. Mocand ${ }^{1,14}$, J. J. Mohr ${ }^{6,11,26}$, T. E. Montroy ${ }^{27}$, S. S. Murray ${ }^{9}$,

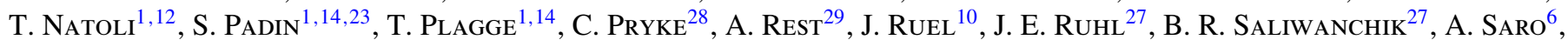

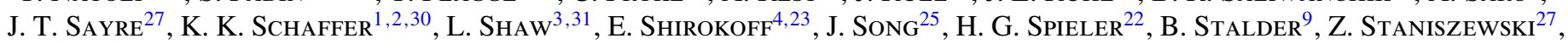
A. A. Stark ${ }^{9}$, K. Story ${ }^{1,12}$, C. W. Stubbs ${ }^{9,10}$, R. Suhada ${ }^{6,11}$, A. van Engelen ${ }^{3}$, K. VAnderlinde ${ }^{3}$, J. D. Vieira $^{1,12,23}$,

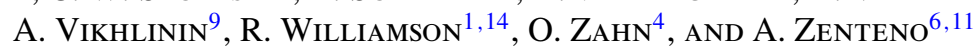

\footnotetext{
${ }^{1}$ Kavli Institute for Cosmological Physics, University of Chicago, 5640 South Ellis Avenue, Chicago, IL 60637, USA; bbenson@ kicp.uchicago.edu

${ }^{2}$ Enrico Fermi Institute, University of Chicago, 5640 South Ellis Avenue, Chicago, IL 60637, USA

${ }^{3}$ Department of Physics, McGill University, 3600 Rue University, Montreal, Quebec H3A 2T8, Canada

${ }^{4}$ Department of Physics, University of California, Berkeley, CA 94720, USA

${ }^{5}$ University of Chicago, 5640 South Ellis Avenue, Chicago, IL 60637, USA

${ }^{6}$ Department of Physics, Ludwig-Maximilians-Universität, Scheinerstr. 1, D-81679 München, Germany

${ }^{7}$ MIT Kavli Institute for Astrophysics and Space Research, Massachusetts Institute of Technology, 77 Massachusetts Avenue, Cambridge, MA 02139, USA

${ }^{8}$ National Center for Supercomputing Applications, University of Illinois, 1205 West Clark Street, Urbana, IL 61801, USA

${ }^{9}$ Harvard-Smithsonian Center for Astrophysics, 60 Garden Street, Cambridge, MA 02138, USA

${ }^{10}$ Department of Physics, Harvard University, 17 Oxford Street, Cambridge, MA 02138, USA

${ }^{11}$ Excellence Cluster Universe, Boltzmannstr. 2, D-85748 Garching, Germany

12 Department of Physics, University of Chicago, 5640 South Ellis Avenue, Chicago, IL 60637, USA

${ }^{13}$ Department of Physics, University of Missouri, 5110 Rockhill Road, Kansas City, MO 64110, USA

${ }^{14}$ Department of Astronomy and Astrophysics, University of Chicago, 5640 South Ellis Avenue, Chicago, IL 60637, USA

${ }^{15}$ Argonne National Laboratory, 9700 S. Cass Avenue, Argonne, IL 60439, USA

${ }^{16}$ NIST Quantum Devices Group, 325 Broadway Mailcode 817.03, Boulder, CO 80305, USA

${ }_{17}$ Departamento de Astronoma y Astrofsica, PUC Casilla 306, Santiago 22, Chile

${ }^{18}$ Department of Astronomy, University of Florida, Gainesville, FL 32611, USA

${ }^{19}$ Department of Astrophysical and Planetary Sciences and Department of Physics, University of Colorado, Boulder, CO 80309, USA

${ }^{20}$ Department of Space Science, VP62, NASA Marshall Space Flight Center, Huntsville, AL 35812, USA

${ }^{21}$ Department of Physics, University of California, One Shields Avenue, Davis, CA 95616, USA

${ }^{22}$ Physics Division, Lawrence Berkeley National Laboratory, Berkeley, CA 94720, USA

${ }^{23}$ California Institute of Technology, 1200 E. California Blvd., Pasadena, CA 91125 , USA

${ }^{14}$ Steward Observatory, University of Arizona, 933 North Cherry Avenue, Tucson, AZ 85721, USA

${ }^{25}$ Department of Physics, University of Michigan, 450 Church Street, Ann Arbor, MI 48109, USA

${ }^{26}$ Max-Planck-Institut für extraterrestrische Physik, Giessenbachstr. D-85748 Garching, Germany

${ }^{27}$ Physics Department and CERCA, Case Western Reserve University, 10900 Euclid Ave., Cleveland, OH 44106, USA

${ }^{28}$ Physics Department, University of Minnesota, 116 Church Street S.E., Minneapolis, MN 55455, USA

${ }^{29}$ Space Telescope Science Institute, 3700 San Martin Dr., Baltimore, MD 21218, USA

${ }^{30}$ Liberal Arts Department, School of the Art Institute of Chicago, 112 S Michigan Ave, Chicago, IL 60603, USA

${ }^{31}$ Department of Physics, Yale University, P.O. Box 208210, New Haven, CT 06520-8120, USA Received 2011 December 22; accepted 2012 December 17; published 2013 January 17
}

\begin{abstract}
We use measurements from the South Pole Telescope (SPT) Sunyaev-Zel'dovich (SZ) cluster survey in combination with X-ray measurements to constrain cosmological parameters. We present a statistical method that fits for the scaling relations of the SZ and X-ray cluster observables with mass while jointly fitting for cosmology. The method is generalizable to multiple cluster observables, and self-consistently accounts for the effects of the cluster selection and uncertainties in cluster mass calibration on the derived cosmological constraints. We apply this method to a data set consisting of an SZ-selected catalog of 18 galaxy clusters at $z>0.3$ from the first $178 \mathrm{deg}^{2} \mathrm{of} \mathrm{the} 2500 \mathrm{deg}^{2}$ SPT-SZ survey, with 14 clusters having X-ray observations from either Chandra or XMM-Newton. Assuming a spatially flat $\Lambda$ CDM cosmological model, we find the SPT cluster sample constrains $\sigma_{8}\left(\Omega_{m} / 0.25\right)^{0.30}=0.785 \pm 0.037$. In combination with measurements of the cosmic microwave background (CMB) power spectrum from the SPT and the seven-year Wilkinson Microwave Anisotropy Probe data, the SPT cluster sample constrains $\sigma_{8}=0.795 \pm 0.016$ and $\Omega_{m}=0.255 \pm 0.016$, a factor of 1.5 improvement on each parameter over the CMB data alone. We consider several extensions beyond the $\Lambda$ CDM model by including the following as free parameters: the dark energy equation of state $(w)$, the sum of the neutrino masses $\left(\Sigma m_{v}\right)$, the effective number of relativistic species $\left(N_{\text {eff }}\right)$, and a primordial non-Gaussianity $\left(f_{\mathrm{NL}}\right)$. We find that adding the SPT cluster data significantly improves the constraints on $w$ and $\Sigma m_{v}$ beyond those found when using measurements of the CMB, supernovae, baryon acoustic oscillations, and the Hubble constant. Considering each extension independently, we best constrain $w=-0.973 \pm 0.063$ and the sum of neutrino masses $\Sigma m_{v}<0.28 \mathrm{eV}$ at $95 \%$ confidence, a factor of 1.25 and 1.4 improvement, respectively,
\end{abstract}


over the constraints without clusters. Assuming a $\Lambda \mathrm{CDM}$ model with a free $N_{\text {eff }}$ and $\Sigma m_{v}$, we measure $N_{\text {eff }}=$ $3.91 \pm 0.42$ and constrain $\Sigma m_{v}<0.63 \mathrm{eV}$ at $95 \%$ confidence. We also use the SPT cluster sample to constrain $f_{\mathrm{NL}}=$ $-220 \pm 317$, consistent with zero primordial non-Gaussianity. Finally, we discuss the current systematic limitations due to the cluster mass calibration, and future improvements for the recently completed $2500 \mathrm{deg}^{2} \mathrm{SPT}_{\mathrm{SZ}} \mathrm{survey}$. The survey has detected $\sim 500$ clusters with a median redshift of $\sim 0.5$ and a median mass of $\sim 2.3 \times 10^{14} M_{\odot} h^{-1}$ and, when combined with an improved cluster mass calibration and existing external cosmological data sets will significantly improve constraints on $w$.

Key words: cosmic background radiation - cosmology: observations - galaxies: clusters: general - large-scale structure of universe

Online-only material: color figures

\section{INTRODUCTION}

Clusters of galaxies are the most massive collapsed objects in the universe. Their abundance is sensitive to multiple cosmological parameters, in particular the matter density, the amplitude of the matter power spectrum, and the dark energy equation of state (e.g., Wang \& Steinhardt 1998; Haiman et al. 2001; Holder et al. 2001). Measurements of the cluster abundance that extend to higher redshifts become sensitive to dark energy through its effect on the growth of structure. This makes cluster abundance measurements an important systematic test of the standard dark energy paradigm, because they are affected by dark energy in a fundamentally different way than distance-redshift-based tests, such as from Type Ia supernovae ( $\mathrm{SNe}$ ) and baryon acoustic oscillations (BAOs). For the same reason, cluster abundance measurements also constrain different cosmological parameter combinations than distance-based tests, and their combination can break parameter degeneracies and achieve tighter constraints than either method alone (e.g., Linder \& Jenkins 2003).

Recently, there has been significant theoretical and experimental progress in efforts to use clusters as cosmological probes. Large-volume numerical simulations have calibrated a "universal" cluster mass function over a broad range of cosmologies at a level better than current experimental uncertainties (e.g., Jenkins et al. 2001; Warren et al. 2006; Tinker et al. 2008; Bhattacharya et al. 2011). Numerical simulations have also led to a better understanding of systematic biases in cluster mass estimates derived from a broad range of cluster observables (e.g., Kravtsov et al. 2006; Jeltema et al. 2008; Stanek et al. 2010; Becker \& Kravtsov 2011). Measurements of the cluster abundance using optical, X-ray, and SZ selection methods have been used to place competitive constraints on cosmology and dark energy parameters (e.g., Vikhlinin et al. 2009b; Mantz et al. 2010c; Rozo et al. 2010; Vanderlinde et al. 2010; Sehgal et al. 2010). Currently, the most precise dark energy constraints from clusters are derived from X-ray-selected samples which use the $\mathrm{X}$-ray emission from the hot intra-cluster gas as a tracer of the total mass in the cluster. X-ray observables, particularly the gas mass and inferred pressure, tend to correlate with cluster mass with low scatter, independent of the dynamical state of the cluster or the details of non-gravitational physics in clusters (e.g., Kravtsov et al. 2006).

Hot intra-cluster gas also causes a spectral distortion in the cosmic microwave background $(\mathrm{CMB})$ in the direction of clusters from inverse Compton scattering, a phenomenon known as the Sunyaev-Zel'dovich (SZ) effect (Sunyaev \& Zel'dovich 1972). The surface brightness of the SZ effect is redshift independent and largest at millimeter $(\mathrm{mm})$ wavelengths. The integrated SZ effect from a cluster is effectively measuring the cluster pressure, and is an observable that is expected to have comparably low scatter with mass to the best X-ray observables (Nagai et al. 2007; Shaw et al. 2008; Stanek et al. 2010). Therefore, an mm-wavelength SZ survey with sufficient angular resolution is expected to provide clean, mass-limited catalogs out to high redshift, probing the regime where the cluster abundance is most sensitive to dark energy's effect on the growth rate of structure (Carlstrom et al. 2002).

Recently, the first SZ cluster catalogs from three surveys have been released: the South Pole Telescope (SPT; Staniszewski et al. 2009; Vanderlinde et al. 2010; Williamson et al. 2011), the Atacama Cosmology Telescope (Marriage et al. 2011), and the Planck satellite (Planck Collaboration et al. 2011). However, even with only $\sim 10-20$ clusters, the cosmological constraints from these surveys have been limited by the systematic uncertainty in the cluster mass calibration (Vanderlinde et al. 2010; Sehgal et al. 2011). X-ray surveys (Vikhlinin et al. 2009b; Mantz et al. 2010c) have achieved tighter constraints by adopting variations of the following calibration strategy: calibrating X-ray observable-mass relations using X-ray hydrostatic mass estimates of relaxed clusters, applying this calibration to a larger sample of relaxed and unrelaxed clusters, and verifying the overall mass calibration from other methods, particularly from weaklensing measurements. In this work, we apply a similar strategy to the SPT-SZ survey using the cluster sample from Vanderlinde et al. (2010, hereafter V10), by incorporating an externally calibrated X-ray observable-mass relation and X-ray measurements of the V10 sample in order to present improved cosmological constraints.

This paper is organized as follows. In Section 2, we describe the relevant SZ, X-ray, and optical data, analysis methods, and the external cosmological data sets used in this work. In Section 3, we describe and implement a self-consistent cosmological analysis using SZ and X-ray observations of the SPT cluster sample that simultaneously constrains cosmology and the relevant SZ and X-ray cluster scaling relations while accounting for the SPT cluster selection function. In Section 4, we discuss the constraints on a $\Lambda$ CDM cosmological model from the SPT cluster sample, and compare our results to the constraints from observations of the $\mathrm{CMB}$ power spectrum. In Section 5, we consider extensions to the $\Lambda \mathrm{CDM}$ model by including the following as free parameters: dark energy equation of state, the sum of the neutrino masses, the effective number of relativistic species, and a primordial non-Gaussianity. We report the relative improvements using the SPT data to constrain each extension. In Sections 6 and 7, we discuss the limiting systematics and implications for applying this method to the larger SPT cluster sample.

In this paper, unless otherwise specified, the cluster mass will refer to $M_{500}$, the mass enclosed within a spherical radius, $r_{500}$, where the cluster's mean matter density is 500 times the 
critical density of the universe at the observed cluster redshift. The critical density is $\rho_{\text {crit }}(z)=3 H^{2}(z) / 8 \pi G$, where $H(z)$ is the Hubble parameter.

\section{DATA AND OBSERVATIONS}

\subsection{Cluster Data and Observations}

The cluster sample used in this work is a sub-sample of a SZ-selected catalog from the SPT that was described in V10. The V10 catalog consisted of 21 clusters selected by their SPT significance from $178 \mathrm{deg}^{2}$ of sky surveyed by the SPT in 2008. As in V10, we use only the 18 clusters at $z>0.3$ for the cosmological results in this work. The optical and X-ray properties of this catalog have been described previously in High et al. (2010) and Andersson et al. (2011), hereafter $\mathrm{H} 10$ and A11, respectively. In this section, we summarize the V10, H10, and A11 data sets, analysis, and results used in this work. We also report additional spectroscopic redshift and X-ray measurements for several clusters.

\subsubsection{SZ Observations and the Cluster Sample}

The $10 \mathrm{~m}$ diameter SPT is an mm-wavelength telescope designed to conduct a large-area survey with low noise and $\sim 1$ arcmin angular resolution. The SPT receiver consists of a 960 element bolometer array that is sensitive in three bands, at 95, 150, and $220 \mathrm{GHz}$. Details of the telescope and receiver can be found in Padin et al. (2008), Carlstrom et al. (2011), and Dobbs et al. (2012). The primary goal of the SPT survey is to search for clusters of galaxies via the SZ effect in a $2500 \mathrm{deg}^{2}$ survey that was completed in 2011 November.

The first cosmological constraints from the SPT cluster survey were reported in V10, with an accompanying cluster catalog. These results were derived from SPT $150 \mathrm{GHz}$ observations of $178 \mathrm{deg}^{2}$ observed in 2008, from two approximately equal area fields centered at right ascension (R.A.) $5^{\mathrm{h}} 30^{\mathrm{m}}$, declination (decl.) $-55^{\circ}$ and R.A. $23^{\mathrm{h}} 30^{\mathrm{m}}$, decl. $-55^{\circ}$. Cluster candidates were identified in the SPT maps by using a matched spatial filter technique (Haehnelt \& Tegmark 1996; Melin et al. 2006). In brief, the SPT maps are filtered in Fourier space to optimize the detection of cluster-like objects using a source template constructed from a $\beta$-model of variable angular size. This is done while accounting for the expected signals from the dominant sources of astrophysical contamination, instrumental and atmospheric noise, and the effects of the SPT beam and timestream filtering. Candidate galaxy clusters were assigned an SPT significance, $\xi$, defined as the highest signal to noise across all filter scales.

V10 used simulations to characterize the SZ selection function and the scaling between $\xi$ and cluster mass. Simulated SZ maps were generated from large-volume dark matter simulations (Shaw et al. 2009) using the semi-analytic gas model of Bode et al. (2007). The gas model was calibrated to match the observed X-ray scaling relations for low-redshift $(z<0.25)$ clusters. The cluster selection was characterized by applying the matched filter to multiple sky realizations that included the dominant astrophysical components (primary and lensed CMB, thermal SZ, and point sources), instrumental and atmospheric noise, and the SPT filtering. These simulations found that at $\xi>5$, the SPT catalog was expected to be $\sim 95 \%$ pure. This result is consistent with optical follow-up which found optical cluster counterparts to 21 of the 22 candidates above this threshold. The 21 optically confirmed clusters had a median redshift of $z=0.74$, and the sample was predicted to be nearly $100 \%$ complete above a mass threshold of $M_{500} \sim 6 \times 10^{14} h^{-1} M_{\odot}$ at $z=0.6$. The simulations were also used to put conservative priors on the relationship between SPT significance and mass in the V10 cosmological analysis. Even with only 18 clusters, the improvement in the cosmological constraints was limited by the assumed systematic uncertainty on the normalization of the relationship between SPT significance and mass.

The full cluster catalog used in this work is given in Table 1. For each cluster, we report the name, position, redshift, and the SZ and X-ray observables, where the latter assumes a default cosmology. We note that the only SZ product needed for the cosmological analysis described in Section 3 is the SZ observable $\xi$. In this work we improve the cosmological constraints relative to $\mathrm{V} 10$ by reducing the uncertainty on the relationship between SPT significance and mass through inclusion of X-ray observables which have an observable-mass relation that has been externally calibrated, as described in Vikhlinin et al. (2009a) and summarized in Section 3.1.1.

\subsubsection{Optical Redshifts}

Redshifts of the SPT clusters were measured through a combination of optical photometry and spectroscopy. The majority of the observations and data analyses are identical to those in $\mathrm{H} 10$, to which we refer the reader for a more detailed description. Relative to $\mathrm{H} 10$, we include spectroscopic redshift measurements for seven additional clusters, which we briefly describe here. All cluster redshifts are given in Table 1.

Optical counterparts and photometric redshifts were measured from a combination of imaging from the Blanco Cosmology Survey (see Ngeow et al. 2009) and targeted observations using the Magellan telescopes. Optical images were searched for red sequence galaxies within a $2^{\prime}$ radius of the SPT candidate location. A cluster was identified through an excess of red sequence galaxies relative to the background, and the photometric redshift was estimated by fitting a red sequence model. The redshift uncertainty varies over the sample; however, it is typically $\Delta z /(1+z) \sim 0.03$.

For 15 of the 18 clusters, we have also measured spectroscopic redshifts, which we use for the cluster's redshift when measured. For eight of the clusters we use the spectroscopic redshifts as reported in H10, which were measured using the Low Dispersion Survey Spectrograph (LDSS3) on the Magellan Clay $6.5 \mathrm{~m}$ telescope. For SPT-CL J0546-5345, we use the redshift reported in Brodwin et al. (2010), measured using multi-slit spectroscopy with the Inamori Magellan Areal Camera and Spectrograph (IMACS) on the Magellan Baade $6.5 \mathrm{~m}$ telescope. Finally, there are six clusters that have new spectroscopic redshifts, which we report in this work for the first time in Table 1 . These redshifts were measured with a combination of IMACS and GMOS on Gemini South, and the details of the data and analysis will be described in S. Bocquet et al. (in preparation).

\subsubsection{X-Ray Observations}

X-ray observations were obtained using Chandra and XMM-Newton for 14 of the clusters in Table 1 . The majority of the X-ray observations, data reduction, and analyses are the same as described by $\mathrm{A} 11$, to which we refer the reader for a more detailed description. Relative to A11, we include new Chandra observations for five clusters, and re-run the X-ray analysis for the five clusters with new optical spectroscopic redshifts, one of which also had new Chandra observations. In this section, we summarize the X-ray observations and results, and 
Table 1

The SPT $178 \mathrm{deg}^{2}$ Cluster Catalog and Observables

\begin{tabular}{|c|c|c|c|c|c|c|}
\hline Object Name & $\begin{array}{l}\text { R.A. } \\
\text { (deg) }\end{array}$ & $\begin{array}{l}\text { decl. } \\
\text { (deg) }\end{array}$ & Photo-z & Spec- $z$ & $\xi$ & $\begin{array}{c}Y_{X} \\
\left(10^{14} M_{\odot} \mathrm{keV}\right)\end{array}$ \\
\hline SPT CL J0509-5342 & 77.336 & -53.705 & $0.47(4)$ & 0.463 & 6.61 & $4.3 \pm 0.8$ \\
\hline SPT-CL J0511-5154 & 77.920 & -51.904 & $0.74(5)$ & $\ldots$ & 5.63 & $\ldots$ \\
\hline SPT-CL J0521-5104 & 80.298 & -51.081 & $0.72(5)$ & $\cdots$ & 5.45 & $\cdots$ \\
\hline SPT-CL J0528-5300 & 82.017 & -53.000 & $0.75(5)$ & 0.765 & 5.45 & $1.6 \pm 0.5^{\mathrm{b}}$ \\
\hline SPT-CL J0533-5005 & 83.398 & -50.092 & $0.83(5)$ & 0.881 & 5.59 & $1.0 \pm 0.4^{\mathrm{b}}$ \\
\hline SPT-CL J0539-5744 & 85.000 & -57.743 & $0.77(5)$ & $\ldots$ & 5.12 & $\ldots$ \\
\hline SPT-CL J0546-5345 & 86.654 & -53.761 & $1.16(6)$ & $1.067^{\mathrm{a}}$ & 7.69 & $4.8 \pm 0.8^{b}$ \\
\hline SPT-CL J0551-5709 & 87.902 & -57.156 & $0.41(4)$ & 0.423 & 6.13 & $1.9 \pm 0.4^{\mathrm{b}}$ \\
\hline SPT-CL J0559-5249 & 89.925 & -52.826 & $0.66(4)$ & 0.611 & 9.28 & $6.4 \pm 0.8$ \\
\hline SPT-CL J2301-5546 & 345.469 & -55.776 & $0.78(5)$ & $0.748^{\mathrm{a}}$ & 5.19 & $\ldots$ \\
\hline SPT-CL J2331-5051 & 352.958 & -50.864 & $0.55(4)$ & 0.571 & 8.04 & $3.5 \pm 0.6$ \\
\hline SPT-CL J2332-5358 & 353.104 & -53.973 & $0.32(3)$ & $0.403^{\mathrm{a}}$ & 7.30 & $6.1 \pm 0.8^{b}$ \\
\hline SPT-CL J2337-5942 & 354.354 & -59.705 & $0.77(5)$ & 0.781 & 14.94 & $8.5 \pm 1.7$ \\
\hline SPT-CL J2341-5119 & 355.299 & -51.333 & $1.03(5)$ & 0.998 & 9.65 & $4.7 \pm 1.0$ \\
\hline SPT-CL J2342-5411 & 355.690 & -54.189 & $1.08(6)$ & $1.074^{\mathrm{a}}$ & 6.18 & $1.4 \pm 0.3^{\mathrm{b}}$ \\
\hline SPT-CL J2355-5056 & 358.955 & -50.937 & $0.35(4)$ & $0.320^{\mathrm{a}}$ & 5.89 & $2.2 \pm 0.4^{b}$ \\
\hline SPT-CL J2359-5009 & 359.921 & -50.160 & $0.76(5)$ & $0.774^{\mathrm{a}}$ & 6.35 & $1.8 \pm 0.4^{\mathrm{b}}$ \\
\hline SPT-CL J0000-5748 & 0.250 & -57.807 & $0.74(5)$ & $0.701^{\mathrm{a}}$ & 5.48 & $4.2 \pm 1.6^{\mathrm{b}}$ \\
\hline
\end{tabular}

Notes. $\xi$ is the maximum signal to noise of the SPT detection obtained over the set of filter scales for each cluster. The cluster positions in R.A. and decl. are given in degrees and refer to the center of the SZ brightness in the SPT map filtered at the preferred scale to maximize the signal to noise. We give the estimated photometric redshift and spectroscopic redshifts, where available. To be consistent with A11, $Y_{X}$ is calculated assuming a preferred $\Lambda \mathrm{CDM}$ cosmology using $W M A P 7+\mathrm{BAO}+H_{0}$ data with $\Omega_{M}=0.272, \Omega_{\Lambda}=0.728$, and $H_{0}=70.2 \mathrm{~km} \mathrm{~s}^{-1} \mathrm{Mpc}^{-1}$ (Komatsu et al. 2011). In Sections 4 and 5, $Y_{X}$ is recalculated as a function of cosmology and scaling relations for each step in the Markov chain.

a New spectroscopic redshift since V10.

${ }^{\mathrm{b}}$ Updated $Y_{X}$ since A11.

describe additional analyses required to incorporate the X-ray measurements in the cosmological analysis.

Summarizing A11, 15 of the 16 highest $\xi$ clusters from V10 were targeted for X-ray observations, however, in this work, we use only the 14 clusters at $z>0.3$. Of these, 12 were observed with Chandra and four clusters were observed with XMM-Newton. Two clusters were observed by both Chandra and XMM-Newton, and for these clusters only the Chandra data were included in the analysis. From the data, the X-ray observables, $M_{g}, T_{X}$, and $Y_{X}$, were measured in a manner identical to Vikhlinin et al. (2009a), where $M_{g}$ is the gas mass within $r_{500}, T_{X}$ is the core-excised $X$-ray temperature in an annulus between $0.15-1.0 \times r_{500}$, and $Y_{X} \equiv M_{g} T_{X}$. We solved for each observable and $r_{500}$ iteratively, to maintain consistency with their respective observable-mass relations. Since A11, five of the clusters have new spectroscopic redshift measurements. For these clusters we repeat the A11 reduction and analysis using the new redshifts, and give the updated results in Table 7.

Five of the clusters from A11 have had additional Chandra observations, which we include in this work. In Table 8, we list these clusters, the Chandra observation IDs, and the improvement in exposure time and cluster source counts adding the new observations. We repeat the A11 reduction and analysis to derive new constraints on the X-ray observables, which are given in Table 7. For these results, relative to A11, we use more recent Chandra analysis software (CIAO 4.3) and calibration files (CALDB 4.3.3). We find that the new Chandra calibration files typically change $Y_{X}$ by $<5 \%$. This is a level below the assumed mass-normalization uncertainty that we assign in Section 3.1.1.

For the cosmological analysis in this work, described in Section 3, we need to calculate the X-ray observables as a function of cosmology and scaling relation parameters. To do this, we derive density and temperature profiles for all 14 clusters with X-ray data. We calculate $T_{X}(r)$ and $M_{g}(r)$ (for the calculation of $\left.Y_{X}(r)\right)$ from the X-ray observations of each cluster assuming a reference cosmology, where $r$ corresponds to a physical radius in the cluster and the profiles are defined to return the cluster observable within $r$. The reference cosmology is chosen to match A11; a preferred $\Lambda$ CDM cosmology using $W M A P 7+\mathrm{BAO}+H_{0}$ data with $\Omega_{M}=0.272, \Omega_{\Lambda}=0.728$, and $H_{0}=70.2 \mathrm{~km} \mathrm{~s}^{-1} \mathrm{Mpc}^{-1}$ (Komatsu et al. 2011). For three of the clusters with the lowest X-ray photon counts, the $T_{X}(r)$ profiles have jumps which appear unphysical. For this reason, we have assumed a functional form of $T_{X}(r)$ that, in combination with the measured $M_{g}(r)$, matches the pressure profile from Arnaud et al. (2010) and is normalized to give the measured $Y_{X}$ assuming the reference cosmology. When considering the 11 clusters with well-behaved temperature profiles, we find that our cosmological results in Section 4 negligibly change when assuming either the functional form of $T_{X}(r)$, or the profile derived from the data. Therefore, we consider this approximation valid for this work.

\subsection{External Cosmological Data Sets}

In addition to the SPT cluster data set, we incorporate several external cosmological data sets, including measurements of the CMB power spectrum $(\mathrm{CMB})$, the Hubble constant $\left(H_{0}\right)$, BAOs, Type Ia SNe, and big bang nucleosynthesis (BBN). We will use these abbreviations when referring to these data sets, and will use several different combinations of them in our analysis and results. Below we give references and a brief description of each external data set. Also, when discussing our results in Section 4 and onward, we will define the $\mathrm{SPT}_{\mathrm{CL}}$ data set as the combination of the SPT-SZ data, optical redshift, and X-ray measurements described in Section 2.1. 
We use measurements of the CMB power spectrum from the seven-year Wilkinson Microwave Anisotropy Probe data release (WMAP7; Larson et al. 2011) and $790 \mathrm{deg}^{2}$ of sky observed with the SPT (Keisler et al. 2011). Following Keisler et al. (2011), ${ }^{32}$ we fit the CMB data to a model including primary CMB anisotropy plus three nuisance parameters that model "foreground" signals detectable in the SPT data. We use lowredshift measurements of $H_{0}$ from the Hubble Space Telescope (Riess et al. 2011), which we include as a Gaussian prior of $H_{0}=73.8 \pm 2.4 \mathrm{~km} \mathrm{~s}^{-1} \mathrm{Mpc}^{-1}$. We use measurements of the BAO feature using Sloan Digital Sky Survey and 2dFGRS data (Percival et al. 2010). The BAO constraints have been applied as a measurement of $r_{s} / D_{V}(z=0.2)=0.1905 \pm$ 0.0061 and $r_{s} / D_{V}(z=0.35)=0.1097 \pm 0.0036$; where $r_{s}$ is the comoving sound horizon size at the baryon drag epoch, $D_{V}(z) \equiv\left[(1+z)^{2} D_{A}^{2}(z) c z / H(z)\right]^{1 / 3}, D_{A}(z)$ is the angular diameter distance, and $H(z)$ is the Hubble parameter. The inverse covariance matrix given in Equation (5) of Percival et al. (2010) is used for the BAO measurements. We use measurements of the luminosity distances of Type Ia SNe from the Union 2 compilation of 557 SNe (Amanullah et al. 2010), and include their treatment of systematic uncertainties. Finally, we use a BBN prior from measurements of the abundances of ${ }^{4} \mathrm{He}$ and deuterium (Kirkman et al. 2003), which we include as a Gaussian prior of $\Omega_{b} h^{2}=0.022 \pm 0.002$.

\section{COSMOLOGICAL ANALYSIS}

In this section, we outline the cosmological analysis method for the SPT cluster data set, including the calculation of the cosmological likelihood and the assumed parameterization for the cluster mass-observable relations. This implementation allows for self-consistent constraints on cosmology and the cluster scaling relations, i.e., the cluster mass calibration, by simultaneously varying the cluster mass-observable relations and cosmological parameters using a Markov Chain Monte Carlo (MCMC) technique. The method is generalizable in a way that can include additional cluster observables from other data sets (e.g., weak-lensing shear, optical velocity dispersions). We have incorporated our calculation of the SPT cluster likelihood into the CosmoMC code ${ }^{33}$ of Lewis \& Bridle (2002) to compute its joint likelihood with the external cosmological data sets.

\subsection{Scaling Relation Parameterization}

$$
\text { 3.1.1. X-Ray: } Y_{X}-M_{500}
$$

Following Vikhlinin et al. (2009b), we use $Y_{X}$ as an X-ray proxy for cluster mass, $M_{500}$. We assume a $Y_{X}-M_{500}$ relation of the form

$$
\frac{M_{500}}{10^{14} M_{\odot} h^{-1}}=\left(A_{X} h^{3 / 2}\right)\left(\frac{Y_{X}}{3 \times 10^{14} M_{\odot} \mathrm{keV}}\right)^{B_{X}} E(z)^{C_{X}}
$$

parameterized by the normalization $A_{X}$, the slope $B_{X}$, the redshift evolution $C_{X}$, where $E(z) \equiv H(z) / H_{0}$, and a log-normal scatter $D_{X}$ on $Y_{X}$. Relative to the form of this equation in Vikhlinin et al. (2009b), we have multiplied the right-hand side by an extra factor of $h$, so that the cluster mass $M_{500}$, is in units of $M_{\odot} h^{-1}$ to match the $\zeta-M_{500}$ relation in Section 3.1.4. For our cosmological analysis, we assume Gaussian priors on the scaling

\footnotetext{
32 http://pole.uchicago.edu/public/data/keisler11

$33 \mathrm{http}: / /$ cosmologist.info/cosmomc/
}

relation parameters, which we list in Table 2. The priors are motivated by constraints from X-ray measurements by Vikhlinin et al. (2009a) and simulations, which we describe below.

Vikhlinin et al. (2009a) constrained the $Y_{X}-M_{500}$ relation using a combination of $X$-ray and weak-lensing measurements. Initially, X-ray observations of a low-redshift $(z<0.3)$ sample of 17 relaxed clusters were used to constrain the $Y_{X}-M_{500}$ relation, using cluster total mass estimates assuming the gas was in hydrostatic equilibrium. This was done for a relaxed cluster sample to minimize biases in the mass estimates from non-thermal pressure support. For example, Nelson et al. (2012) found that bulk motions induced after a cluster's merger can contribute up to $30 \%$ of the total pressure, but quickly decays to $\sim 10 \%-15 \%$ as the cluster relaxes. The dynamical state of the cluster is not expected to significantly affect the $Y_{X}-M_{500}$ relation, which is expected from simulations to be relatively unchanged between relaxed and unrelaxed clusters (Kravtsov et al. 2006; Poole et al. 2007; Rasia et al. 2011), with Kravtsov et al. (2006) putting an upper limit on the systematic offset between their normalization of $3 \%$. This conclusion is supported by recent measurements comparing $Y_{X}$ and weak-lensing mass estimates for a sample of 50 massive clusters (Mahdavi et al. 2012). From the X-ray measurements alone, Vikhlinin et al. (2009a) obtained best-fit values of $A_{X}=5.77 \pm 0.20$ and $B_{X}=0.57 \pm 0.03$, where the uncertainties are statistical only.

Vikhlinin et al. (2009a) then used weak-lensing measurements to estimate the uncertainty of the $A_{X}$ calibration by comparing $Y_{X}$-based and weak-lensing mass estimates for two sets of observations of low-redshift $(z<0.3)$ clusters. First, they compared the weak-lensing mass estimates in Hoekstra (2007) to their own $Y_{X}$-based mass estimates for the 10 clusters at $z<0.3$ that also had sufficient Chandra observations to measure $Y_{X}$. They combined this result with measurements from Zhang et al. (2008), which similarly compared weak-lensing and $Y_{X}$-based mass estimates for a sample of 19 clusters at $z<0.3$, to estimate a $1 \sigma$ uncertainty of $9 \%$ on the Chandra mass scale calibration. Adding this uncertainty in quadrature with the statistical uncertainty from the $Y_{X}-M_{500}$ constraints in Vikhlinin et al. (2009a), we assign a Gaussian prior of $A_{X}=5.77 \pm 0.56$, which translates to a $10 \%$ uncertainty in the cluster mass calibration at $z=0$. In Section 3.1.2, we revisit the accuracy of this calibration by comparing to several more recent weak-lensing results.

We assume a Gaussian prior of $C_{X}=-0.4 \pm 0.2$, consistent with self-similar evolution and a $50 \%$ uncertainty. In Section 3.1.3, we discuss the motivation for this prior in detail. In brief, it is motivated by the largest deviation from selfsimilar redshift evolution from a set of three hydrodynamical cluster simulations (Kravtsov et al. 2006; Short et al. 2010; Fabjan et al. 2011) designed to study the redshift evolution of the $Y_{X}-M_{500}$ relation assuming a broad range of astrophysical processes, and hence should explore a reasonable range of possible evolution scenarios. The uncertainty in $C_{X}$ effectively adds a redshift-dependent uncertainty to the cluster mass calibration. For example, at $z=0.74$ and $z=1.074$, the median and maximum redshift for the clusters in this work, the uncertainty in $C_{X}$ adds an additional $8 \%$ and $11 \%$ uncertainty, respectively, to the cluster mass calibration.

We assume a Gaussian prior of $D_{X}=0.12 \pm 0.08$, which we truncate below 0.02 and where $D_{X}=0.12$ corresponds to a $12 \% \log$-normal scatter in $Y_{X}$ for a given mass. This scatter has been measured to have values ranging from $0 \%$ to 12\% (Vikhlinin et al. 2009a; Mantz et al. 2010a). Analogous 
Table 2

Parameter Table

\begin{tabular}{|c|c|c|c|}
\hline Type & Symbol & Meaning & Gaussian Prior \\
\hline Scaling & $A_{\mathrm{SZ}}$ & $\zeta$-mass normalization & $5.58 \pm 1.67$ \\
\hline Relation & $B_{\mathrm{SZ}}$ & $\zeta$-mass slope & $1.32 \pm 0.26$ \\
\hline \multirow[t]{7}{*}{ Parameters } & $C_{\mathrm{SZ}}$ & $\zeta$-mass redshift evolution & $0.87 \pm 0.44$ \\
\hline & $D_{\mathrm{SZ}}$ & Log-normal scatter in $\zeta$ & $0.24 \pm 0.16$ \\
\hline & $A_{X}$ & $Y_{X}$-mass normalization & $5.77 \pm 0.56$ \\
\hline & $B_{X}$ & $Y_{X}$-mass slope & $0.57 \pm 0.03$ \\
\hline & $C_{X}$ & $Y_{X}$-mass redshift evolution & $-0.40 \pm 0.20$ \\
\hline & $D_{X}$ & Log-normal scatter in $Y_{X}$ & $0.12 \pm 0.08$ \\
\hline & $\rho$ & Correlated scatter between $\zeta$ and $Y_{X}$ & Uniform:(0.02, 0.98) \\
\hline Primary & $\Omega_{c} h^{2}$ & Dark matter density & \\
\hline Cosmology & $\Omega_{b} h^{2}$ & Baryon density & \\
\hline \multirow[t]{4}{*}{ Parameters } & $100 \Theta_{s}$ & Angular scale of the sound horizon at last scattering & \\
\hline & $n_{s}$ & Scalar tilt of power spectrum & \\
\hline & $10^{9} \Delta_{R}^{2}$ & Scalar amplitude of power spectrum & \\
\hline & $\tau$ & Optical depth to reionization & \\
\hline Extension & $w$ & Dark energy equation of state & \\
\hline Cosmology & $f_{v}$ & Fraction of dark matter in the form of neutrinos, $\Sigma m_{v}=94 \mathrm{eV}\left(f_{v} \Omega_{c} h^{2}\right)$ & \\
\hline \multirow[t]{2}{*}{ Parameters } & $N_{\text {eff }}$ & The effective number of relativistic species & \\
\hline & $f_{\mathrm{NL}}$ & Primordial non-Gaussianity parameter & \\
\hline Derived & $\sigma_{8}$ & Matter fluctuations on $8 \mathrm{Mpc}$ scales at $z=0$ & \\
\hline Cosmology & $\Omega_{m}$ & Total matter density & \\
\hline Parameters & $h$ & $h \equiv H_{0} / 100 \mathrm{~km} \mathrm{~s}^{-1} \mathrm{Mpc}^{-1}$, where $H_{0}$ is the Hubble constant at $z=0$ & \\
\hline
\end{tabular}

to Vikhlinin et al. (2009b), for our cosmological analysis we have chosen a prior centered on a value which is consistent with simulations (e.g., Kravtsov et al. 2006), but additionally assumed a larger uncertainty than the range typically found in simulations.

In Section 3.2, we describe how the assumed $Y_{X}-M_{500}$ relation is included in our cosmological likelihood model and translate the predicted cluster number density to observable space. In Section 6, we estimate the uncertainty in the cosmological constraints from the assumed priors on the parameters in the $Y_{X}-M_{500}$ relation. These results quantify the relative importance of each parameter, and also can be used to estimate the impact of either systematic offsets or improved constraints in the $Y_{X}-M_{500}$ relation on the cosmological constraints.

\subsubsection{Testing the Normalization of the $Y_{X}-M_{500}$ Relation}

In this work, our cluster mass calibration is primarily determined through the priors assumed for the $Y_{X}-M_{500}$ relation, which were motivated by a combination of X-ray and weak-lensing measurements as described in Vikhlinin et al. (2009a) and summarized in Section 3.1.1. Since the publication of Vikhlinin et al. (2009a), there have been several weak-lensing results (e.g., Hoekstra et al. 2012; Applegate et al. 2012; High et al. 2012) that could affect the normalization of the $Y_{X}-M_{500}$ relation, and our cluster mass calibration. We revisit our assumptions in light of these results.

Our original prior on the mass normalization of the $Y_{X}-M_{500}$ relation $\left(A_{X}\right)$ was based on the results of Vikhlinin et al. (2009a), which constrained the $Y_{X}-M_{500}$ relation using $\mathrm{X}$-ray observations of a low-redshift $(z<0.3)$ sample of 17 relaxed clusters to estimate $Y_{X}$ and the cluster total mass by assuming the gas was in hydrostatic equilibrium. In part, they tested the accuracy of this calibration by comparing weaklensing and $Y_{X}$-based mass estimates for a sample of 10 clusters at $z<0.3$ with sufficient Chandra observations to measure $Y_{X}$ and that also had published weak-lensing mass estimates in Hoekstra (2007). From this comparison, they found that $M_{500, \mathrm{WL}}=(1.01 \pm 0.11) \times M_{500, Y_{X}}$.

More recently, the weak-lensing masses from Hoekstra (2007) have twice been updated by the same author (Mahdavi et al. 2008; Hoekstra et al. 2012). We repeat the analysis by Vikhlinin et al. (2009a), comparing the $Y_{X}$ and weak-lensingbased mass estimates for the same 10 clusters at $z<0.3$ considered in that work, but now using the most recent weak-lensing mass estimates from Hoekstra et al. (2012). Doing this, we find a best-fit relation of $M_{500, \mathrm{wL}}=(0.98 \pm 0.08) \times M_{500, Y_{X}}$, consistent with our previous assumed calibration and uncertainty on $A_{X}$.

Independently, a series of papers (von der Linden et al. 2012; Kelly et al. 2012; Applegate et al. 2012) described the methodology and results for a project to measure weaklensing masses for a sample of 51 massive clusters, with an estimated systematic uncertainty of $\sim 7 \%$. Applegate et al. (2012) compared their weak-lensing masses to multiple results in the literature, including Mahdavi et al. (2008), with the two works having eight overlapping clusters. The masses from Mahdavi et al. (2008) were found to be lower by a mean factor of 0.88 , with no formal uncertainty quoted. Given the mean uncertainty of the individual cluster masses in Mahdavi et al. (2008), $\sim 30 \%-35 \%$, we expect this uncertainty to be $\sim 35 \% / \sqrt{8} \approx 12 \%$, and the constraint on the mean ratio of cluster masses in Mahdavi et al. (2008) to Applegate et al. (2012) to be $0.88 \pm 0.12$. We can translate this result to Hoekstra et al. (2012) by comparing the published masses between Mahdavi et al. (2008) and Hoekstra et al. (2012). Doing this, we find that the latter were smaller by a mean factor of 1.05 . We therefore expect that the weak-lensing masses in Applegate et al. (2012) are larger than the weak-lensing masses in Hoekstra et al. (2012) by a factor of $1.19 \pm 0.16$, and therefore larger than the $Y_{X}$-based masses in Vikhlinin et al. (2009a) by a factor of $1.17 \pm 0.16$.

Also of note, Applegate et al. (2012) found that the weaklensing masses in Okabe et al. (2010) were lower by a mean 
factor of 0.77 , and noted several reasons why the analysis in Okabe et al. (2010) would systematically bias their weak-lensing mass estimates low. Using the weak-lensing mass estimates from Okabe et al. (2010), Marrone et al. (2012) measured a $Y_{\mathrm{SZ}}-M_{500}$ scaling relation and found a $\sim 30 \%$ offset in mass for fixed $Y_{\mathrm{SZ}}$ compared to Andersson et al. (2011), which estimated cluster masses using the same $Y_{X}-M_{500}$ relation used in this work. However, the same team behind Okabe et al. (2010) is currently revising their weak-lensing mass estimates to address some of the issues pointed out in the literature. Preliminary results indicate that their revised masses are consistent with Applegate et al. (2012) and the resultant $Y_{\mathrm{SZ}}-M_{500}$ relation is consistent with Andersson et al. (2011) (Marrone 2012, private communication). For these reasons, we do not include the results of Okabe et al. (2010) in reconsidering the normalization of the $Y_{X}-M_{500}$ relation.

Recently, High et al. (2012) presented weak-lensing mass estimates for five SPT-selected clusters, using a nearly identical weak-lensing analysis to Hoekstra et al. (2012). High et al. (2012) compared their results to SZ-based mass estimates from Reichardt et al. (2012b), which used the same methodology and mass calibration as assumed in this work, determined primarily by the same $Y_{X}-M_{500}$ relation. High et al. (2012) found that the spherical weak-lensing masses measured within $r_{500, S Z}$ were on average larger than the SZ-based mass estimates by a factor of $1.07 \pm 0.18$.

Therefore, all three weak-lensing results from Hoekstra et al. (2012), Applegate et al. (2012), and High et al. (2012) are statistically consistent with each other, and our assumed calibration of the $Y_{X}-M_{500}$ relation. If we take a weighted average of the three, we infer that the weak-lensing data favor larger cluster masses by a factor of $1.03 \pm 0.07$ relative to our assumed $Y_{X}-M_{500}$ calibration, for which we had assigned a $10 \%$ uncertainty in the cluster mass calibration at $z=0$. Therefore, our assumed calibration of the $Y_{X}-M_{500}$ relation is both consistent and of comparable uncertainty to an updated analysis using the results from more recent weak-lensing results.

\subsubsection{The Redshift Evolution of the $Y_{X}-M_{500}$ Relation}

There are relatively few observational constraints on the normalization of the $Y_{X}-M_{500}$ relation at $z>0.3$, so our prior on the redshift evolution of the $Y_{X}-M_{500}$ relation, $C_{X}$, in Section 3.1.1, is based on predictions from simulations. We consider three sets of simulations: Kravtsov et al. (2006), Short et al. (2010), and Fabjan et al. (2011). Each work presents results of hydrodynamical simulations of clusters that include several effects that evolve with redshift during cluster formation, including the increased frequency of cluster mergers and larger non-thermal pressure support at higher redshift, and also include a variety of astrophysical feedback processes.

Kravtsov et al. (2006) used a high-resolution adaptive-mesh refinement code that incorporated radiative cooling, and a range of astrophysical phenomena, including: dissipation-less dynamics of dark matter, gas-dynamics, star formation, metal enrichment and thermal feedback due to Type II and Type Ia $\mathrm{SNe}$, self-consistent advection of metals, metallicity-dependent radiative cooling, and UV heating due to cosmological ionizing background. They are the highest-resolution simulations we consider, but also have the fewest number of clusters (16). They find that the redshift evolution of the $Y_{X}$ observable closely matches the self-similar prediction, and provide an upper limit of $<5 \%$ for the change in the mass normalization at a fixed $Y_{X}$ between $z=0$ and 0.6 .
Fabjan et al. (2011) describe the results of high-resolution hydrodynamical simulations of 140 clusters with mass $>5 \times$ $10^{13} M_{\odot} h^{-1}$ using the TreePM-SPH GADGET code (Springel et al. 2005). In order to test the robustness of mass proxies against changes in the physical processes included in the simulation, they simulate a sub-sample of 18 clusters in seven different flavors to study the effects of: thermal conduction, viscosity, cooling and star formation, galactic winds, and active galactic nuclei. As a general result, they find the $Y_{X}-M_{500}$ relation to be the least sensitive scaling relation to variations in assumed physics, with its redshift evolution always being very close to the self-similar prediction. The largest deviation from self-similar redshift evolution is in their non-radiative standard viscosity simulation, which implies a $7 \%$ change in the mass normalization at fixed $Y_{X}$ between $z=0$ and 1.0.

Short et al. (2010) are based on the Millennium Gas Simulation (Springel 2005), which were re-simulations of the original $500 \mathrm{Mpc} h^{-1}$ Millennium simulation with three different physical treatments: shock heating driven by gravity only (GO), cooling and preheating (PH), and a feedback run using a semianalytic model of galaxy formation (FO). The FO model most closely matches the measured $Y_{X}-M_{500}$ normalization and redshift evolution when comparing to measurements of real clusters. The FO model also has the smallest redshift evolution, with a deviation from self-similar evolution of only $2 \%$ in mass at fixed $Y_{X}$ between $z=0$ and 1.0. The PH model is also reasonably consistent with the measurements, however it predicts the most significant deviation from self-similar evolution, even when compared to the results from Kravtsov et al. (2006) and Fabjan et al. (2011). The PH model finds a deviation from selfsimilar that translates to $12 \%$ change in the mass normalization at fixed $Y_{X}$ between $z=0$ and 1.0 .

In the above simulations, the largest deviation from selfsimilar redshift evolution is the PH model of Short et al. (2010), which found a $12 \%$ change in mass normalization at $z=1.0$. In Section 3.1.1, the assumed prior on the redshift evolution of the $Y_{X}-M_{500}$ relation, $C_{X}=-0.4 \pm 0.2$, translated to a $11 \%$ uncertainty in mass normalization for the largest redshift cluster in this work at $z=1.074$. Therefore, we consider our assumed prior on $C_{X}$ to be consistent with the simulations above, which represent a range of possible redshift evolutions from a broad range of possible astrophysical effects.

$$
\text { 3.1.4. SZ: } \zeta-M_{500}
$$

The SPT detection significance, $\xi$, is used to both select the cluster sample and as a cluster mass proxy. As discussed in V10 and Melin et al. (2006), primary CMB temperature anisotropies provide a source of astrophysical confusion that add significant uncertainty in determining the integrated Comptonization, $Y_{\mathrm{SZ}}$, for a given cluster. In V10, the SPT significance, $\xi$, was introduced as an alternative SZ mass proxy, and has been used in this way in subsequent SPT cluster works, including A11 and Williamson et al. (2011). In this section, we review the relationship between SPT significance and cluster mass, and discuss specifics related to its implementation in this work. In Section 3.2, we will describe how the assumed relationship between SPT significance and cluster mass is included in our cosmological model, including how the cluster selection based on SPT significance affects our predicted cluster number density.

In V10, the relationship between SPT significance and cluster mass was characterized through simulated SZ cluster maps. The maps were generated following the method of Shaw et al. (2009), using a semi-analytic gas model of Bode et al. (2007) applied to 
halos in a large-volume dark matter simulation. The simulations were used to characterize and remove a maximization bias in $\xi$, from maximizing the SPT significance across the cluster position and core radius. This led to the introduction of the unbiased significance, $\zeta$ : defined as the average detection signal to noise across many realizations, evaluated at the preferred position and filter scale of that cluster as determined by fitting the cluster in the absence of noise. The simulations found that $\zeta$ can be related to $\langle\xi\rangle$ through the relation

$$
\zeta=\sqrt{\langle\xi\rangle^{2}-3}
$$

at $\xi>2$. Allowing the position and cluster core radius to vary effectively adds three degrees of freedom to the fit with $\xi$ analogous to a $\chi^{2}$, and $\langle\xi\rangle$ relating to $\xi$ by a Gaussian scatter of unit width. Simulations have been used to verify that these approximations introduce negligible bias or scatter compared to the Poisson noise of the sample.

We assume a $\zeta-M_{500}$ relation of the form

$$
\zeta=A_{\mathrm{SZ}}\left(\frac{M_{500}}{3 \times 10^{14} M_{\odot} h^{-1}}\right)^{B_{\mathrm{SZ}}}\left(\frac{E(z)}{E(0.6)}\right)^{C_{\mathrm{SZ}}}
$$

parameterized by the normalization $A_{\mathrm{SZ}}$, the slope $B_{\mathrm{SZ}}$, the redshift evolution $C_{\mathrm{SZ}}$, and a $\log$-normal scatter, $D_{\mathrm{SZ}}$, on $\zeta$. In $\mathrm{V} 10$, the form of this equation was motivated by the expected self-similar relationship between $Y_{\mathrm{SZ}}$ and cluster mass. In this way, the relationship between SPT significance and cluster mass can be predicted directly in the limiting cases of either spatially unresolved or resolved clusters. For the SPT survey, most clusters lie somewhere between these two limits, depending on the angular size and redshift of each cluster. In V10, the SPT significance-mass relation was fit assuming three different simulations: two with different levels of astrophysical feedback, and a third that assumed cluster pressure profiles that matched the "universal" form in Arnaud et al. (2010). The range in the fit values were then used to motivate conservative priors on the scaling relation parameters. We note that a similar exercise was repeated in $\mathrm{A} 11$ to fit a $\zeta-Y_{\mathrm{SZ}}$ relation.

In this work, to be consistent with the $Y_{X}-M_{500}$ relation, we are defining the cluster mass as $M_{500}$, the mass in a spherical radius, $r_{500}$, within which the density is equal to 500 times the critical density of the universe at the cluster redshift. This change has motivated a change in the redshift evolution term from $(1+z)$ to $E(z)$, because of the expected self-similar scaling between $Y_{\mathrm{SZ}}$ and $M_{500}$ (e.g., Kravtsov et al. 2006). In addition, we include a correlated scatter between $\zeta$ and $Y_{X}$ with a correlation coefficient $\rho$, which we allow to uniformly vary between 0.02 and 0.98 , but away from 0 and 1 for numerical reasons. Analogous to V10, we refit the simulations for the $\zeta-M_{500}$ relation in Equation (3) and we give the Gaussian priors in Table 2. The fractional uncertainty on each parameter matches V10, except for the lognormal scatter, for which we allow a larger uncertainty in this work.

The priors on the $\zeta-M_{500}$ scaling parameters do not significantly affect the cosmological constraints in this work (see Section 6). The SPT and X-ray observations typically constrain the $\zeta-M_{500}$ relation better than the simulation-based priors because of the relatively tight external observational constraints on the $Y_{X}-M_{500}$ relation, which are reflected in the priors discussed in Section 3.1.1. For example, the $\zeta-M_{500}$ and $Y_{X}-M_{500}$ relations have priors that correspond to a $30 \%$ and $10 \%$ uncertainty on their normalization, respectively. Therefore, SPT and X-ray observations of even a few clusters can, in principle, improve upon the $30 \%$ prior on the $\zeta-M_{500}$ normalization.

As also discussed in Section 3.1.2, High et al. (2012) compared SPT and weak-lensing-based mass estimates for five SPT-selected clusters from Reichardt et al. (2012b), and found them to agree with an overall accuracy of $\sim 18 \%$, limited by the statistical uncertainty of the weak-lensing measurements. This result suggests that our current priors on the $\zeta-M_{500}$ relation are relatively conservative. In Section 6, we discuss the relative importance of our assumed priors on the $\zeta-M_{500}$ relation and estimate their effect on the derived cosmological constraints.

\subsection{Likelihood Model}

The analysis method employed in this work closely mirrors the one presented by V10 with extensions to incorporate the $\mathrm{X}$-ray data. In V10, the parameter space was explored through importance sampling of pre-existing WMAP MCMC chains. In this work, we have elected to utilize a full MCMC algorithm. This is accomplished through the use of the CosmoMC analysis package, where we have included the cluster abundance likelihood as an additional module in the CosmoMC likelihood calculation. Among the numerous advantages to this approach is the ability to enforce quantitative convergence criteria as well as the optional inclusion of supplemental data sets.

Each step in the Markov chain selects a new point in the joint cosmological and scaling relation parameter space. Prior to passing these variables to the cluster likelihood evaluation, we use the Code for Anisotropies in the Microwave Background (Lewis et al. 2000) to compute the matter power spectrum at 20 logarithmically spaced redshifts between $0<z<2.5$. The matter power spectra, as well as the proposed scaling relation and relevant cosmological parameters, are the inputs to the cluster likelihood function.

At this point, the analysis follows a similar path to that laid out by V10. First, the matter power spectra and cosmology are used to calculate a mass function based upon the Tinker et al. (2008) prescription, which we calculate for an overdensity of $\Delta=$ $500 \Omega_{m}(z)$, to match our cluster mass definition in Section 3.1. As noted in Tinker et al. (2008), this function predicts the halo abundance as a function of input cosmology across a mass range of $10^{11} h^{-1} M_{\odot} \leqslant M \leqslant 10^{15} h^{-1} M_{\odot}$ and a redshift range of $0 \leqslant z \leqslant 2.5$. Tinker et al. (2008) claim an overall calibration of their mass function to simulations of $\lesssim 5 \%$. Stanek et al. (2010) found that the inclusion of non-gravitational physics can shift the normalization of the mass function by $\sim 10 \%$ along the mass direction. However, this effect is approximately degenerate with an uncertainty between intra-cluster gas observables and mass, which we account for explicitly in our scaling relation uncertainty through Equations (1) and (3).

As in V10, the next step in the analysis is to move the theoretically predicted cluster abundances from their native $M_{500}$ mass space into the observable space for this analysis. V10 define this space by the SZ detection significance, $\xi$, and the optically derived redshift, $z$. This resulted in a two-dimensional surface of predicted cluster abundances in the observable space. In this analysis, we perform a similar transformation, this time including a third dimension, the $\mathrm{X}$-ray parameter $Y_{X}$. This results in a three-dimensional volume of predicted cluster abundances, now as a function of $\xi, Y_{X}$, and $z$.

Using the scaling relations discussed in Section 3.1, the halo mass function is recast as a predicted number density in terms 
Table 3

$\Lambda \mathrm{CDM}$ Constraints

\begin{tabular}{|c|c|c|c|c|}
\hline Parameter & Prior & $\mathrm{SPT}_{\mathrm{CL}}+H_{0}+\mathrm{BBN}$ & $\mathrm{CMB}$ & $\mathrm{CMB}+\mathrm{SPT}_{\mathrm{CL}}$ \\
\hline$A_{\mathrm{SZ}}$ & $5.58 \pm 1.67$ & $5.31 \pm 0.98$ & $\ldots$ & $4.91 \pm 0.71$ \\
\hline$B_{\mathrm{SZ}}$ & $1.32 \pm 0.26$ & $1.39 \pm 0.15$ & $\ldots$ & $1.40 \pm 0.15$ \\
\hline$C_{\mathrm{SZ}}$ & $0.87 \pm 0.44$ & $0.90 \pm 0.34$ & $\ldots$ & $0.83 \pm 0.30$ \\
\hline$D_{\mathrm{SZ}}$ & $0.24 \pm 0.16$ & $0.21 \pm 0.10$ & $\ldots$ & $0.21 \pm 0.09$ \\
\hline$A_{X}$ & $5.77 \pm 0.56$ & $5.69 \pm 0.51$ & $\ldots$ & $5.82 \pm 0.48$ \\
\hline$B_{X}$ & $0.57 \pm 0.03$ & $0.564 \pm 0.029$ & $\ldots$ & $0.563 \pm 0.029$ \\
\hline$C_{X}$ & $-0.40 \pm 0.20$ & $-0.37 \pm 0.16$ & $\ldots$ & $-0.35 \pm 0.16$ \\
\hline$D_{X}$ & $0.12 \pm 0.08$ & $0.14 \pm 0.07$ & $\ldots$ & $0.14 \pm 0.07$ \\
\hline$\rho$ & $(0.02,0.98)$ & $0.52 \pm 0.27$ & $\cdots$ & $0.52 \pm 0.27$ \\
\hline$\Omega_{c} h^{2}$ & $\ldots$ & $0.133 \pm 0.045$ & $0.111 \pm 0.0048$ & $0.109 \pm 0.0032$ \\
\hline$\Omega_{b} h^{2}$ & $\ldots$ & $0.0221 \pm 0.0020$ & $0.0222 \pm 0.0004$ & $0.0223 \pm 0.0004$ \\
\hline $100 \Theta_{s}$ & $\ldots$ & $1.065 \pm 0.041$ & $1.041 \pm 0.0016$ & $1.041 \pm 0.0016$ \\
\hline$n_{s}$ & $(0.944,0.989)$ & $0.966 \pm 0.013$ & $0.965 \pm 0.011$ & $0.967 \pm 0.010$ \\
\hline $10^{9} \Delta_{R}^{2}$ & $\ldots$ & $2.16 \pm 1.30$ & $2.44 \pm 0.10$ & $2.40 \pm 0.08$ \\
\hline$\tau$ & $\cdots$ & $(0.090)$ & $0.086 \pm 0.014$ & $0.087 \pm 0.014$ \\
\hline$\sigma_{8}$ & $\ldots$ & $0.766 \pm 0.062$ & $0.808 \pm 0.024$ & $0.795 \pm 0.016$ \\
\hline$\Omega_{m}$ & $\cdots$ & $0.285 \pm 0.083$ & $0.268 \pm 0.025$ & $0.255 \pm 0.016$ \\
\hline$h$ & $\ldots$ & $0.739 \pm 0.024$ & $0.707 \pm 0.022$ & $0.717 \pm 0.016$ \\
\hline
\end{tabular}

Notes. The marginalized constraints on the scaling relation and primary cosmology parameters from Table 2 , where we report the mean of the likelihood distribution and the $68 \%$ confidence interval about the mean. The priors are Gaussian, except for $\rho$ and $n_{s}$, which are uniform over the range given. The $n_{s}$ prior is only used for the $\mathrm{SPT}_{\mathrm{CL}}+H_{0}+\mathrm{BBN}$ data set.

of $\xi, Y_{X}$, and $z$, which we write as

$\frac{d N\left(\xi, Y_{X}, z \mid \boldsymbol{p}\right)}{d \xi d Y_{X} d z}=\int d M P\left(\xi, Y_{X} \mid M, z, \boldsymbol{p}\right) P(M, z \mid \boldsymbol{p}) \Theta(\xi-5)$,

where $\boldsymbol{p}$ is the set of cosmological and scaling relation parameters, and $\Theta$ is the Heaviside step function. The likelihood function is then given by the Poisson probability:

$$
\begin{aligned}
\ln \mathcal{L}(\boldsymbol{p})= & \sum_{i} \ln \frac{d N\left(\xi_{i}, Y_{X i}, z_{i}, \mid \boldsymbol{p}\right)}{d \xi d Y_{X} d z} \\
& -\int \frac{d N\left(\xi, Y_{X}, z, \mid \boldsymbol{p}\right)}{d \xi d Y_{X} d z} d \xi d Y_{X} d z,
\end{aligned}
$$

where the sum over the $i$ index runs over the SPT cluster catalog. Note that we have neglected a global offset to the log-likelihood.

We compute Equation (4) on a three-dimensional grid that is 200 by 200 by 30 in the $\zeta, Y_{X}$, and $z$ dimensions, respectively. For each value of $Y_{X}$ and $z$ we then convert to the $\xi$ basis by using the $\zeta-\xi$ relation defined in Equation (2), where we also convolve with a unit-width Gaussian in $\xi$ to account for the noise in the SPT measurement.

For each step in the MCMC, we recalculate $Y_{X}$ for each cluster given its $T_{X}(r)$ and $M_{g}(r)$ profiles from Section 2.1.3, so that its calculated $Y_{X}$ is consistent with the $Y_{X}-M_{500}$ relation and $r_{500}$ at that step. To account for this in the cosmological likelihood, we modify the likelihood by adding $\sum_{i} \ln Y_{X i}$ to the right-hand side of Equation (5). For a detailed explanation (see Appendix B). For each cluster, we account for finite measurement errors or missing data in $z$ and $Y_{X}$ by modifying the first term in Equation (5) by marginalizing over the relevant parameter, weighted by either a Gaussian likelihood determined from its uncertainty or a uniform distribution over the allowed range.

From this calculation we obtain a value for the cluster likelihood corresponding to this particular set of cosmological and scaling relation parameters. This value is then returned to
CosmoMC where it may be combined with other likelihood calculations from supplemental data sets and is used in the MCMC step acceptance/rejection computation.

\section{4. $\Lambda$ CDM RESULTS}

We first consider the $\mathrm{SPT}_{\mathrm{CL}}$ data constraints for a spatially flat $\Lambda \mathrm{CDM}$ cosmological model. For this model, we fit 15 parameters: the nine scaling relation parameters and six primary cosmology parameters listed in Table 3. For constraints on any individual parameter, we always quote the mean of the likelihood distribution and the $68 \%$ confidence interval about the mean. The confidence interval reflects uncertainties after marginalizing over all other parameters, and includes systematic uncertainties in the cluster scaling relations and mass calibration, as described in Section 3.1. In this analysis, we use the SPT $\mathrm{CL}_{\mathrm{L}}$ and external cosmological data sets as described in Section 2.

\subsection{Cosmological Constraints}

The $\mathrm{SPT}_{\mathrm{CL}}$ data are not sensitive to all six $\Lambda \mathrm{CDM}$ cosmology parameters. Here and in Section 5.1, when considering the $\mathrm{SPT}_{\mathrm{CL}}$ cosmological constraints without $\mathrm{CMB}$ data, we always include BBN and $H_{0}$ priors, as indicated. For the $\mathrm{SPT}_{\mathrm{CL}}+H_{0}+\mathrm{BBN}$ data set, we also fix the optical depth of reionization, $\tau$, and allow the scalar tilt, $n_{s}$, to vary uniformly between 0.944 and 0.989 , the $95 \%$ confidence range from Keisler et al. (2011) assuming a $\Lambda$ CDM model. However, we note that the $\mathrm{SPT}_{\mathrm{CL}}$ cosmological constraints vary negligibly over this range of $n_{s}$. As noted in Section 2.2, whenever we refer to the $\mathrm{SPT}_{\mathrm{CL}}$ data, we are implicitly referring to the combined SPT-SZ data, optical redshift, and X-ray measurements described in Section 2.1.

In Figure 1, we show the constraints on the $\sigma_{8}$ and $\Omega_{m}$ parameters for the individual and combined $\mathrm{SPT}_{\mathrm{CL}}$ and $\mathrm{CMB}$ data sets. In Table 3, we give the marginalized constraints for the cosmological and scaling relation parameters. The latter will be discussed further in Sections 4.2 and 6. In a $\Lambda$ CDM cosmology, the SPT $\mathrm{CL}_{\text {data }}$ are most sensitive to $\sigma_{8}$ and $\Omega_{m}$. 


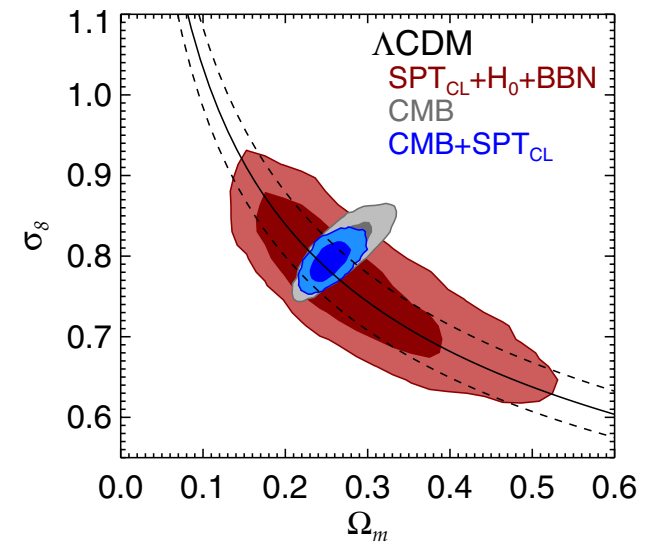

Figure 1. Assuming a $\Lambda \mathrm{CDM}$ cosmology, the two-dimensional marginalized constraints on $\sigma_{8}$ and $\Omega_{m}$. Contours show the $68 \%$ and $95 \%$ confidence regions for the $\mathrm{SPT}_{\mathrm{CL}}+H_{0}+\mathrm{BBN}$ (red), CMB (gray), and $\mathrm{CMB}+\mathrm{SPT}_{\mathrm{CL}}$ (blue) data sets. The black lines are the best-fit constraint (solid) and $68 \%$ confidence region (dashed) for the combination of parameters that the $\mathrm{SPT}_{\mathrm{CL}}+H_{0}+\mathrm{BBN}$ data set best constrains: $\sigma_{8}\left(\Omega_{m} / 0.25\right)^{0.30}=0.785 \pm 0.037$.

(A color version of this figure is available in the online journal.)

The number of clusters increases with either parameter, so the cluster abundance data effectively constrain a product of the two. We find that the $\mathrm{SPT}_{\mathrm{CL}}+H_{0}+\mathrm{BBN}$ constraints are well approximated as $\sigma_{8}\left(\Omega_{m} / 0.25\right)^{0.30}=0.785 \pm 0.037$, which we show in Figure 1 by the solid and dashed lines. Combining the $\mathrm{SPT}_{\mathrm{CL}}$ and CMB data, we constrain $\sigma_{8}=0.795 \pm 0.016$ and $\Omega_{m}=0.255 \pm 0.016$, a factor of 1.5 improvement on each over the constraints from the $\mathrm{CMB}$ alone.

The SPT $_{\mathrm{CL}}$ constraints are consistent with results using optical and X-ray-selected cluster samples. Recently, Rozo et al. (2010) compared the cluster constraints from several different methods, and found generally good agreement and comparable constraints. It is typical for cluster-based constraints to be quoted in terms of the product of $\sigma_{8}$ and $\Omega_{m}$ to an exponent which varies depending on the mass scale of the cluster sample. One example for comparison is Vikhlinin et al. (2009b), who constrained $\sigma_{8}\left(\Omega_{m} / 0.25\right)^{0.47}=0.813 \pm 0.027$. For typical $\Lambda$ CDM model constraints of $\Omega_{m} \sim 0.25-0.30$, this agrees well with our result.

\subsection{Scaling Relation Constraints}

In Figure 2, we show the relationship between $\xi$ and $Y_{X}$ for the 14 clusters with $X$-ray observations, overplotted with the expected distribution of clusters and the best-fit relation determined using the $\mathrm{CMB}+\mathrm{SPT}_{\mathrm{CL}}$ data from Section 4.1. The combination of the steep mass function and SZ selection yields a distribution of clusters visibly offset from the best-fit scaling relation, an effect often referred to as Eddington bias. We note that our cosmological analysis method described in Section 3.2 explicitly accounts for the SZ selection and therefore Eddington bias. We also predict the expected distribution of clusters in the $\xi-Y_{X}$ plane assuming the best-fit cosmology and scaling relation parameters, and applying a comparable selection as was used for the SPT X-ray follow-up $(z>0.3$ and $\xi>5.45)$. The predicted 14.2 clusters is consistent with the 14 detected. In Figure 2, we overplot the effective $68 \%$ and $95 \%$ confidence region in the $\xi-Y_{X}$ plane where we would expect to find these clusters. Qualitatively, we find good agreement between the observed and predicted cluster distribution.

In Table 3, we give the constraints on the $Y_{X}-M_{500}$ and $\zeta-M_{500}$ scaling relations using the $\mathrm{SPT}_{\mathrm{CL}}$ and $\mathrm{CMB}+\mathrm{SPT}_{\mathrm{CL}}$ data sets. Because the $Y_{X}-M_{500}$ relation has significantly tighter

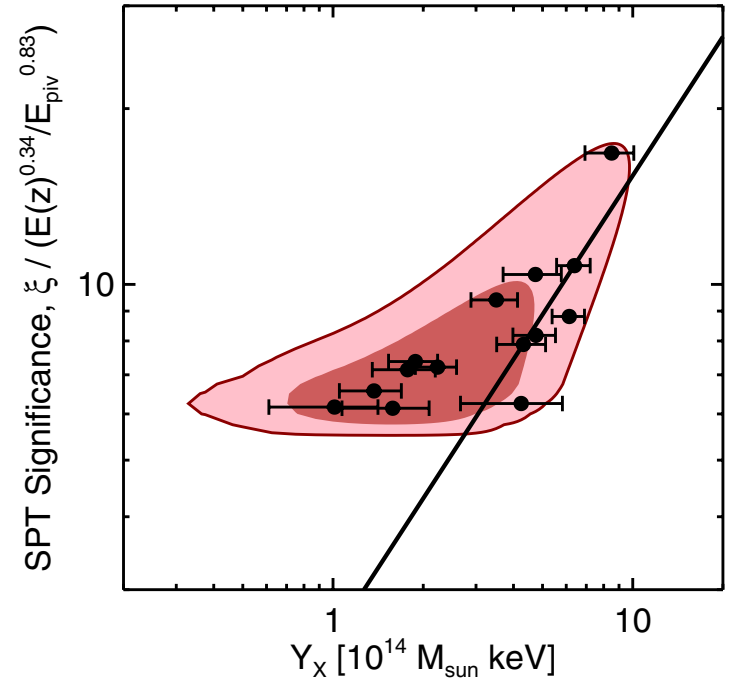

Figure 2. Plot of the SPT significance, $\xi$, vs. the X-ray observable $Y_{X}$ for the 14 SPT clusters with $X$-ray measurements. From the $C M B+S P T_{C L}$ data set fit to a $\Lambda \mathrm{CDM}$ cosmology, we use the best-fit $\zeta-M_{500}$ and $Y_{X}-M_{500}$ scaling relations to calculate the expected form and redshift evolution of the $\xi-Y_{X}$ relation (solid line), where $E_{\text {piv }} \equiv E(z=0.6)$. With the best-fit cosmology parameters, we also predict the effective 68 and $95 \%$ confidence intervals for the expected distribution of clusters in the $\xi-Y_{X}$ plane (red contours). The measured and predicted cluster distribution show qualitatively good agreement.

(A color version of this figure is available in the online journal.)

priors than the $\zeta-M_{500}$ relation, we will not give the $Y_{X}-M_{500}$ constraints for the modified cosmologies presented in Section 5. Similarly, for the parameter $\rho$, the correlated scatter between $\zeta$ and $Y_{X}$, we have virtually no constraining power. In all cases, $\rho$ moves nearly uniformly across the entire allowed range, and has a negligible effect on the cosmological constraints. In Appendix C, we give posterior mass estimates for each cluster using a similar method as described in V10 and briefly reviewed in the Appendix.

If there were a significant discrepancy between the simulation-based prior on the $\zeta-M_{500}$ relation and the observational prior on the $Y_{X}-M_{500}$ relation, we would observe it as an offset between the central value of the $\zeta-M_{500}$ prior and its best-fit value. From the $\mathrm{CMB}+\mathrm{SPT}_{\mathrm{CL}}$ constraints, the largest offset is for $A_{\mathrm{SZ}}$, with a best-fit value of $4.91 \pm 0.71$ compared to the simulation prior of $5.58 \pm 1.67$. An offset in this direction would be consistent with the SZ simulation prior underestimating the mass of a cluster by a factor of $\sim((4.91 \pm 0.71) / 5.58)^{1 / 1.4}=0.91 \pm 0.09$. This result is consistent with preliminary estimates from A11, who estimated this factor to be $0.78 \pm 0.06$. We note that A11 did not marginalize over uncertainties in either the X-ray scaling relation or cosmological parameters, both of which affect this result. The derived offset is also a function of the assumed cosmology. For example, if we assume a $\Lambda$ CDM cosmology with a non-zero neutrino mass, as in Section 5.2, we find a value closer to the simulation prior, $A_{\mathrm{SZ}}=5.39 \pm 0.79$, using the $\mathrm{CMB}+\mathrm{H}_{0}+\mathrm{SPT}_{\mathrm{CL}}$ data set. Therefore, we find no significant inconsistency between the simulation-based prior on the $\zeta-M_{500}$ relation and the observational prior on the $Y_{X}-M_{500}$ relation.

\section{EXTENSIONS TO $\Lambda$ CDM}

In this section, we consider extensions to a spatially flat $\Lambda C D M$ cosmology. For each extension, we also fit the nine scaling relation parameters and six primary cosmology 
Table 4

$w$ CDM Constraints

\begin{tabular}{|c|c|c|c|c|c|}
\hline & & $\mathrm{CMB}$ & $\begin{array}{c}H_{0}+\mathrm{BBN} \\
+\mathrm{SPT}_{\mathrm{CL}} \\
\end{array}$ & $\mathrm{CMB}+\mathrm{BAO}+\mathrm{SNe}$ & $\begin{array}{c}\mathrm{CMB}+\mathrm{BAO}+\mathrm{SNe} \\
+\mathrm{SPT}_{\mathrm{CL}} \\
\end{array}$ \\
\hline Scaling & $A_{\mathrm{SZ}}$ & $\ldots$ & $5.12 \pm 1.36$ & $\ldots$ & $4.75 \pm 0.79$ \\
\hline \multirow[t]{3}{*}{ Parameters } & $B_{\mathrm{SZ}}$ & $\ldots$ & $1.40 \pm 0.15$ & $\ldots$ & $1.41 \pm 0.15$ \\
\hline & $C_{\mathrm{SZ}}$ & $\ldots$ & $0.92 \pm 0.36$ & $\ldots$ & $0.85 \pm 0.29$ \\
\hline & $D_{\mathrm{SZ}}$ & $\ldots$ & $0.22 \pm 0.10$ & $\ldots$ & $0.21 \pm 0.10$ \\
\hline Cosmology & $\sigma_{8}$ & $0.864 \pm 0.120$ & $0.773 \pm 0.088$ & $0.823 \pm 0.040$ & $0.793 \pm 0.028$ \\
\hline \multirow[t]{3}{*}{ Parameters } & $\Omega_{m}$ & $0.244 \pm 0.089$ & $0.293 \pm 0.113$ & $0.279 \pm 0.016$ & $0.273 \pm 0.015$ \\
\hline & $h$ & $0.775 \pm 0.128$ & $0.740 \pm 0.025$ & $0.698 \pm 0.018$ & $0.697 \pm 0.018$ \\
\hline & $w$ & $-1.19 \pm 0.37$ & $-1.09 \pm 0.36$ & $-1.014 \pm 0.078$ & $-0.973 \pm 0.063$ \\
\hline
\end{tabular}

Notes. The marginalized constraints on a subset of the scaling relation and cosmology parameters from Table 2. Scaling relation and primary cosmology parameters not given are still varied in the MCMC and marginalized over for these constraints. We report the mean of the likelihood distribution and the $68 \%$ confidence interval about the mean.

parameters listed in Table 2. We consider four extension cosmologies where we include the following as free parameters: the dark energy equation of state $(w)$, the sum of the neutrino masses $\left(\Sigma m_{v}\right)$, the sum of neutrino masses and the effective number of relativistic species $\left(N_{\text {eff }}\right)$, and a primordial non-Gaussianity $\left(f_{\mathrm{NL}}\right)$. For constraints on any individual parameter, we always quote the mean of the likelihood distribution and the $68 \%$ confidence interval about the mean. The confidence interval will include uncertainties after marginalizing over all other parameters, which includes systematic uncertainties in the cluster scaling relations and mass calibration, as described in Section 3.1. In this analysis, we use the $\mathrm{SPT}_{\mathrm{CL}}$ and external cosmological data sets as described in Section 2.

\section{1. $w C D M$}

The first extension we consider is a wCDM cosmology, a model in which the equation of state of dark energy is a constant $w$. The cluster abundance and the shape of the mass function depend on $w$ through its effect on the growth of structure, or equivalently the redshift evolution of $\sigma_{8}$. The CMB data measure structure at $z \sim 1100$, and therefore require significant extrapolation to predict the cluster abundance in the redshift range of the SPT sample $(0.3<z<1.1)$. Therefore, consistency between the implied $w$ from both data sets is already an important systematic test of dark energy.

In Figure 3, we show the constraints on $w$ and $\sigma_{8}$ using the $\mathrm{CMB}$ and $\mathrm{SPT}_{\mathrm{CL}}+H_{0}+\mathrm{BBN}$ data sets. The likelihood contours have significant overlap, implying that the data are in good agreement. Relative to the CMB, the $\mathrm{SPT}_{\mathrm{CL}}$ data tend to disfavor cosmologies with large $\sigma_{8}$ and more negative $w$. In Table 4, we give marginalized constraints for several cosmological and scaling relation parameters. The SPT $_{\mathrm{CL}}$ data constrain $w=-1.09 \pm 0.36$, and have similar constraining power to the CMB data, for which the constraints have a significant degeneracy between $w$ and $\sigma_{8}$. The $\mathrm{SPT}_{\mathrm{CL}}$ data simultaneously constrain $\sigma_{8}=0.773 \pm 0.088$. This constraint has a factor of $\sim 1.4$ lower uncertainty than that from the CMB data.

\subsection{1. wCDM with BAO and SNe Data Sets}

In this section, we consider the improvement in $w \mathrm{CDM}$ cosmological constraints when adding the $\mathrm{SPT}_{\mathrm{CL}}$ data to the $\mathrm{CMB}, \mathrm{BAO}$, and $\mathrm{SN}$ data sets. In Figure 4, we show the constraints of the combined $\mathrm{CMB}+\mathrm{BAO}+\mathrm{SNe}$ data set, before and after including the $\mathrm{SPT}_{\mathrm{CL}}$ data. The $\mathrm{SPT}_{\mathrm{CL}}$ data most significantly improve the constraints on $\sigma_{8}$ and $w$; reducing the allowed two-dimensional likelihood area by a factor of

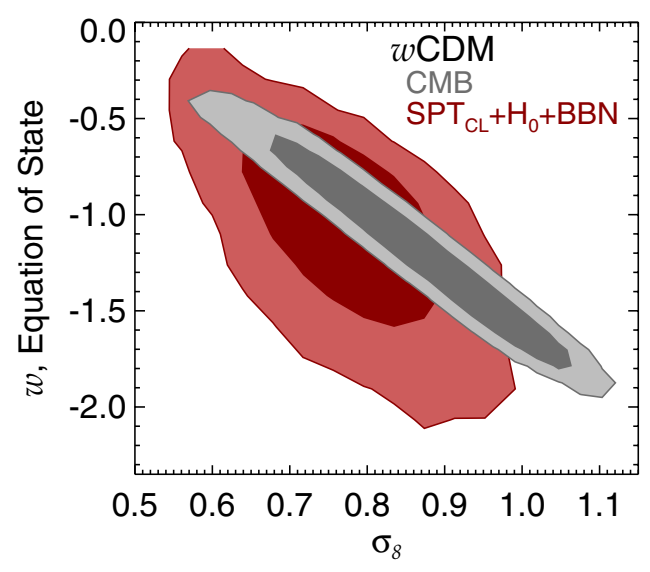

Figure 3. Assuming a $w \mathrm{CDM}$ cosmology, the two-dimensional marginalized constraints on $w$ and $\sigma_{8}$. Contours show the $68 \%$ and $95 \%$ confidence regions for the $\mathrm{SPT}_{\mathrm{CL}}+H_{0}+\mathrm{BBN}$ (red) and $\mathrm{CMB}$ (gray) data sets.

(A color version of this figure is available in the online journal.)

1.8. In Table 4 , we give the marginalized constraints for several parameters before and after the inclusion of the $\mathrm{SPT}_{\mathrm{CL}}$ data. The combined constraints are $w=-0.973 \pm 0.063$ and $\sigma_{8}=0.793 \pm 0.028$, a factor of 1.25 and 1.4 improvement, respectively, over the constraints without clusters. The combined data set also constrains $\Omega_{m}=0.273 \pm 0.015$ and $h=$ $0.697 \pm 0.018$.

The above constraints are consistent with previous clusterbased results (Vikhlinin et al. 2009b; Mantz et al. 2010c; Rozo et al. 2010), which used X-ray and optically selected samples of typically lower redshift clusters. The sensitivity of the SPT $\mathrm{CL}$ cluster data to the amplitude of structure, $\sigma_{8}$, is primarily what gives it the ability to break degeneracies with the distancerelation-based constraints from the $\mathrm{BAO}$ and $\mathrm{SN}$ data sets. We note the slight tension with the $H_{0}$ constraints from Riess et al. (2011) of $h=0.738 \pm 0.024$. While this tension is not significant, it helps to intuitively explain some constraints on neutrino mass in Section 5.2.

\subsection{2. $\zeta-M_{500}$ Constraints}

Given the work of V10 and other cluster results (e.g., Vikhlinin et al. 2009b; Mantz et al. 2010c; Rozo et al. 2010), we expect the cluster mass calibration to be the dominant systematic uncertainty limiting our results. In Figure 5, we show the constraints on $A_{\mathrm{SZ}}$ and $\sigma_{8}$. The $\mathrm{SPT}_{\mathrm{CL}}+H_{0}+\mathrm{BBN}$ data set has a significant degeneracy between its constraints on 


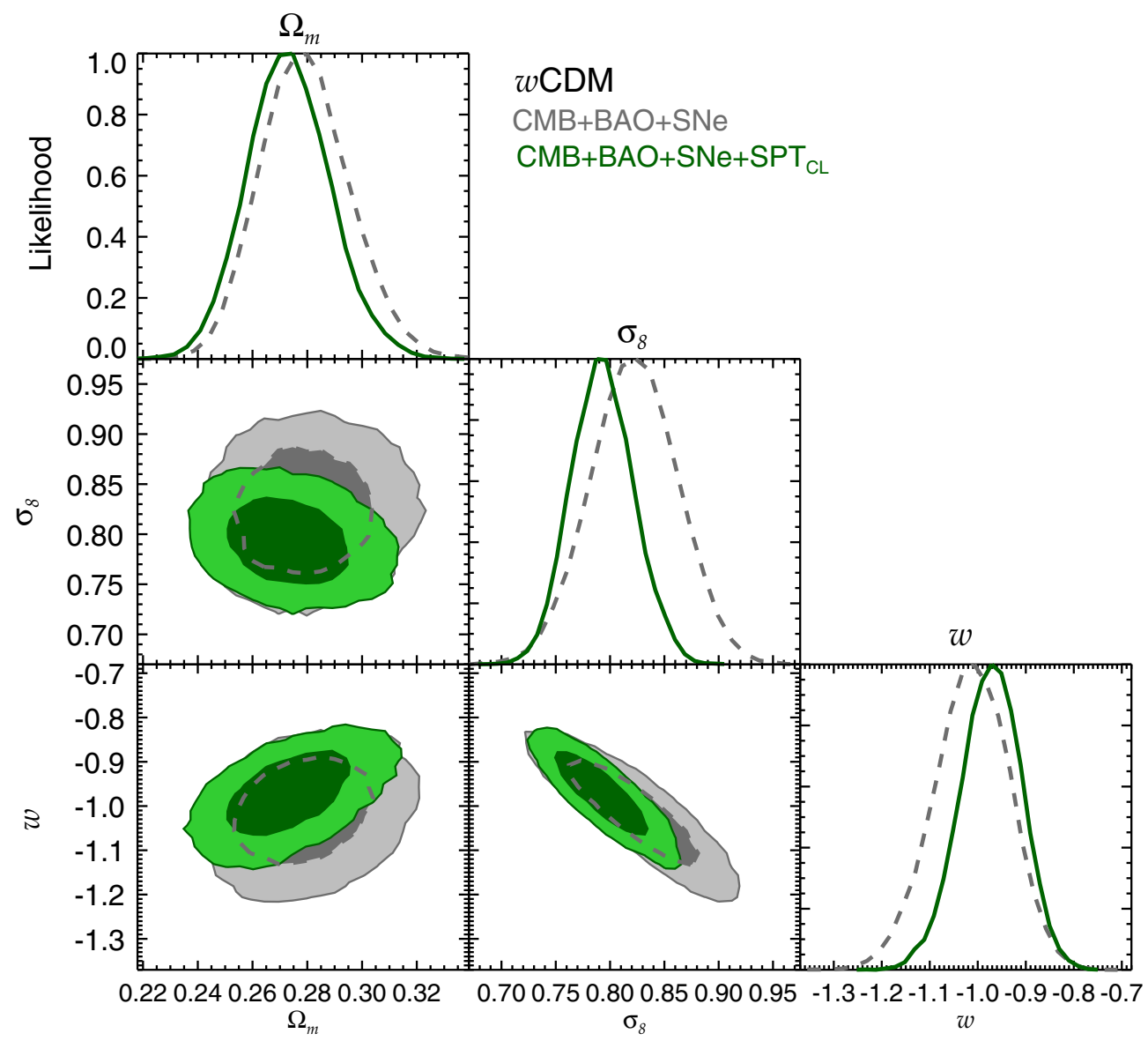

Figure 4. Assuming a $w \mathrm{CDM}$ cosmology, the constraints on $\Omega_{m}, \sigma_{8}$, and $w$. The plots along the diagonal are the one-dimensional marginalized likelihood. The off-diagonal plots are the two-dimensional marginalized constraints showing the $68 \%$ and $95 \%$ confidence regions. We show the constraints for the CMB+BAO+SNe (gray, dashed), and $\mathrm{CMB}+\mathrm{BAO}+\mathrm{SNe}+\mathrm{SPT}_{\mathrm{CL}}$ (green, solid) data sets. The $\mathrm{SPT}_{\mathrm{CL}}$ data improve the constraints on $\sigma_{8}$ and $w$, by factors of 1.4 and 1.25 , respectively.

(A color version of this figure is available in the online journal.)

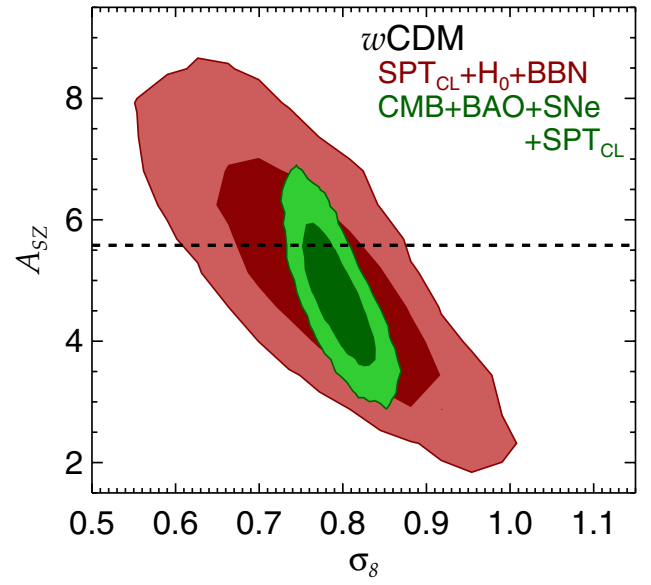

Figure 5. Assuming a $w \mathrm{CDM}$ cosmology, the two-dimensional marginalized constraints on $A_{\mathrm{SZ}}$ and $\sigma_{8}$. Contours show the $68 \%$ and $95 \%$ confidence regions for the $\mathrm{SPT}_{\mathrm{CL}}+H_{0}+\mathrm{BBN}$ (red) and $\mathrm{CMB}+\mathrm{BAO}+\mathrm{SNe}+\mathrm{SPT}$ (green) data sets. The horizontal black dashed line is the center of the theory prior on $A_{\mathrm{SZ}}$.

(A color version of this figure is available in the online journal.)

$A_{\mathrm{SZ}}$ and $\sigma_{8}$. From these data, we constrain the fractional uncertainty, $\delta A_{\mathrm{SZ}} / A_{\mathrm{SZ}}$, to be $27 \%$, which is effectively constrained only by the 14 clusters that have both X-ray and SZ measurements. This constraint is not significantly better than the uncertainty in the simulation based prior of $30 \%$. With enough $\mathrm{X}$-ray observations, we expect the $\zeta-M_{500}$ calibration to be limited by the uncertainty of the $Y_{X}-M_{500}$ relation, because the latter is currently better observationally constrained. In this limit, we would expect a fractional uncertainty on $A_{\mathrm{SZ}}$ of $B_{\mathrm{SZ}} \times\left(\delta A_{X} / A_{X}\right) \sim 14 \%$. The above would suggest that for a $w \mathrm{CDM}$ cosmology we would need X-ray observations of $\sim 50$ clusters, i.e., $\sim 14$ clusters $\times(27 \% / 14 \%)^{2}$, to calibrate $A_{\mathrm{SZ}}$ in terms of mass so that it is not the dominant source of uncertainty. When adding the $\mathrm{BAO}$ and $\mathrm{SN}$ data sets, we improve the constraints on $A_{\mathrm{SZ}}$ to an accuracy of $\sim 16 \%$. However, these data sets are not sensitive to either $A_{\mathrm{SZ}}$ or $\sigma_{8}$, and cannot completely break their degeneracy. In Section 6, we will discuss the systematic uncertainties from this degeneracy on our cosmological constraints in more detail.

\section{2. $\Lambda C D M$ with Massive Neutrinos}

We next consider a $\Lambda \mathrm{CDM}$ cosmology with non-zero neutrino masses. Cosmological measurements are primarily sensitive to the neutrino masses through their effect on structure formation. A massive neutrino additionally affects the CMB power spectrum if it was non-relativistic at the redshift of recombination. For example, if the heaviest neutrino had a mass $\lesssim 0.6 \mathrm{eV}$, it would be relativistic at recombination and therefore would not significantly affect the structure in the CMB (Komatsu et al. 2009). However, as the universe expanded and cooled neutrinos would transition to non-relativistic, and would contribute to $\Omega_{m}$ but not to structure formation below their free streaming scale, implying a lower $\sigma_{8}$ at $z=0$ and fewer clusters. This implies 
Table 5

$\Lambda \mathrm{CDM}+\Sigma m_{v}+N_{\text {eff }}$ Constraints

\begin{tabular}{lccccc}
\hline \hline & & $\mathrm{CMB}+\mathrm{H}_{0}+\mathrm{BAO}$ & $\begin{array}{c}\mathrm{CMB}+\mathrm{H}_{0}+\mathrm{BAO} \\
+\mathrm{SPT}_{\mathrm{CL}}\end{array}$ & $\begin{array}{c}\mathrm{CMB}+\mathrm{H}_{0} \\
+\mathrm{SPT}_{\mathrm{CL}}\end{array}$ & $\begin{array}{c}\mathrm{CMB}+\mathrm{H}_{0}+\mathrm{BAO} \\
+\mathrm{SPT}_{\mathrm{CL}}\end{array}$ \\
\hline Scaling & & $5.26 \pm 0.79$ & $5.39 \pm 0.79$ & $5.01 \pm 0.85$ \\
Parameters & $A_{\mathrm{SZ}}$ & $\ldots$ & $1.39 \pm 0.14$ & $1.39 \pm 0.14$ & $1.41 \pm 0.15$ \\
& $B_{\mathrm{SZ}}$ & $\ldots$ & $0.89 \pm 0.30$ & $0.89 \pm 0.30$ & $0.89 \pm 0.30$ \\
& $C_{\mathrm{SZ}}$ & $\ldots$ & $0.20 \pm 0.09$ & $0.20 \pm 0.10$ & $0.21 \pm 0.10$ \\
\hline Cosmology & $D_{\mathrm{SZ}}$ & $\ldots$ & $0.770 \pm 0.026$ & $0.771 \pm 0.023$ & $0.777 \pm 0.031$ \\
Parameters & $\sigma_{8}$ & $0.761 \pm 0.043$ & $0.272 \pm 0.015$ & $0.260 \pm 0.018$ & $0.284 \pm 0.018$ \\
& $\Omega_{m}$ & $0.275 \pm 0.016$ & $0.701 \pm 0.013$ & $0.712 \pm 0.017$ & $0.727 \pm 0.020$ \\
& $h$ & $0.698 \pm 0.014$ & $0.15 \pm 0.10$ & $0.12 \pm 0.09$ & $0.34 \pm 0.17$ \\
& $\Sigma m_{v}(\mathrm{eV})$ & $<.19 \pm 0.14$ & $<0.33$ & $<0.28$ & $<0.63$ \\
& $\Sigma m_{v}(\mathrm{eV}), 95 \% \mathrm{CL}$ & $<0.45$ & $(3.046)$ & $(3.046)$ & $3.91 \pm 0.42$ \\
\hline
\end{tabular}

Notes. The marginalized constraints on a subset of the scaling relation and cosmology parameters from Table 2. Scaling relation and primary cosmology parameters not given are still varied in the MCMC and marginalized over for these constraints. We report the mean of the likelihood distribution and the $68 \%$ confidence interval about the mean.

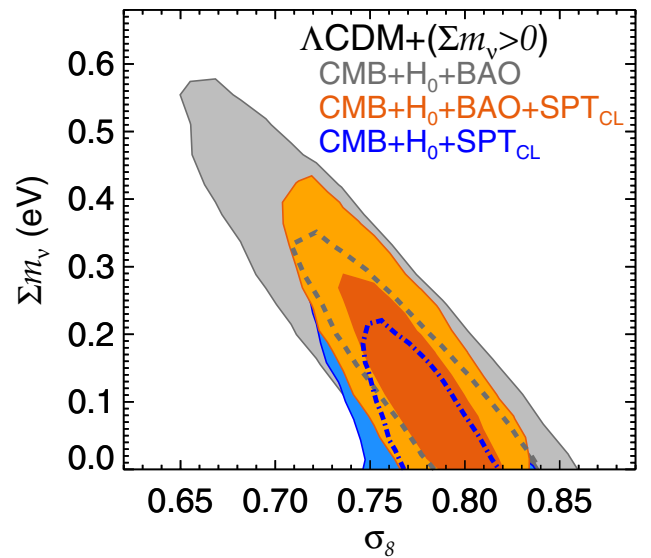

Figure 6. Assuming a $\Lambda \mathrm{CDM}$ cosmology with massive neutrinos, the twodimensional marginalized constraints on $\Sigma m_{v}$ and $\sigma_{8}$. Contours show the $68 \%$ and $95 \%$ confidence regions for the $\mathrm{CMB}+\mathrm{H}_{0}+\mathrm{BAO}$ (gray, dashed), $\mathrm{CMB}+\mathrm{H}_{0}+\mathrm{BAO}+\mathrm{SPT}_{\mathrm{CL}}$ (orange, solid), and $\mathrm{CMB}+\mathrm{H}_{0}+\mathrm{SPT}_{\mathrm{CL}}$ (blue, dot-

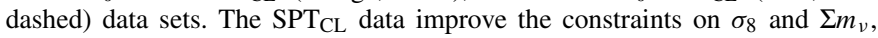
by factors of 1.8 and 1.4, respectively.

(A color version of this figure is available in the online journal.)

that measurements of the CMB power spectrum alone cannot constrain the neutrino mass to significantly less than $0.6 \mathrm{eV}$ per species (i.e., $\Sigma m_{v} \lesssim 1.8 \mathrm{eV}$ ), and the constraints will be significantly degenerate with $\sigma_{8}$. Local measurements of structure break this degeneracy, and significantly improve the neutrino mass constraints.

We also note the significant degeneracy between the CMB power spectrum constraints on $H_{0}$ and $\Sigma m_{v}$. Massive neutrinos affect the amplitude of the early integrated Sachs-Wolfe effect causing a shift of the first peak of the CMB power spectrum toward larger angular scales that can be absorbed by a lower value of $H_{0}$ (Ichikawa et al. 2005). Komatsu et al. (2011) used a combination of $W M A P 7+H_{0}+\mathrm{BAO}$ data to set a limit of $\Sigma m_{v}<0.58$ at $95 \%$ confidence. Following Komatsu et al. (2011), we consider the same combination of data sets to add to the CMB power spectrum measurements, which were chosen because of their insensitivity to systematic errors and their ability to maximally constrain $\Sigma m_{v}$ by breaking the degeneracy with $H_{0}$. We define $\Sigma m_{v}=94 \mathrm{eV}\left(f_{v} \Omega_{c} h^{2}\right)$, where $f_{v}$ is the fraction of dark matter in the form of massive neutrinos.

In Figure 6, we show the constraints on $\sigma_{8}$ and $\Sigma m_{v}$, using the $\mathrm{CMB}+H_{0}+\mathrm{BAO}$ data set, before and after including the
SPT $_{\mathrm{CL}}$ data. In Table 5, we give the marginalized constraints on each parameter. Using the $\mathrm{CMB}+H_{0}+\mathrm{BAO}+\mathrm{SPT}_{\mathrm{CL}}$ data set, we constrain $\Sigma m_{v}<0.33 \mathrm{eV}$ at a $95 \% \mathrm{CL}$, a factor of 1.4 improvement over the constraints without the $\mathrm{SPT}_{\mathrm{CL}}$ data. This improvement is primarily due to the tighter constraints on $\sigma_{8}$ for which the uncertainty decreased by a factor 1.8 . The constraint is lower by excluding the BAO data; using only the $\mathrm{CMB}+H_{0}+\mathrm{SPT}_{\mathrm{CL}}$ data set we constrain $\Sigma m_{v}<0.28 \mathrm{eV}$ at a 95\% CL. These improved constraints can be understood from the $H_{0}$ measurements, as also noted in Section 5.1. The results of Riess et al. (2011) favor a marginally higher $H_{0}$ value than the $\mathrm{CMB}+\mathrm{BAO}$ data. Because of the degeneracy between $\Sigma m_{v}$ and $H_{0}$ in the $\mathrm{CMB}$ constraints, a higher value of $H_{0}$, tends to favor lower values of $\Sigma m_{v}$.

The constraints on $\Sigma m_{v}$ presented here are comparable to other recent results using optically and X-ray-selected cluster samples with similar cosmological data sets (Reid et al. 2010; Mantz et al. 2010b).

\subsubsection{Number of Relativistic Species}

Recent measurements have shown a $\sim 2 \sigma$ preference for increased damping in the tail of the $\mathrm{CMB}$ power spectrum (Dunkley et al. 2011; Keisler et al. 2011). This damping could be caused by several different physical mechanisms, such as a high primordial helium abundance, a running of the scalar spectral index, or additional relativistic species. This last explanation is particularly timely because of recent measurements from atmospheric (Aguilar-Arevalo et al. 2010) and nuclear reactor (Mention et al. 2011) neutrino oscillation experiments that find some evidence for a sterile neutrino species. It has been pointed out that these measurements are most consistent with two sterile neutrinos and $\Sigma m_{v} \gtrsim 1.7 \mathrm{eV}$ (Kopp et al. 2011). Therefore, we consider the joint cosmological constraints on $N_{\text {eff }}$ and $\Sigma m_{v}$ to compare with these terrestrial results.

With only three neutrino species, we would expect $N_{\text {eff }}=$ 3.046 , a value slightly larger than three because of energy injection from electron-positron annihilation at the end of neutrino freeze-out (Dicus et al. 1982; Lopez et al. 1999; Mangano et al. 2005). As $N_{\text {eff }}$ increases, the contribution to the gravitational potential of the additional neutrino perturbations boosts the early growth of dark matter perturbations (Bashinsky $\&$ Seljak 2004), which also increases $\sigma_{8}$ (Hou et al. 2011). As explained in Section 5.2, adding neutrino mass at the levels considered here only affects the low-redshift universe, 


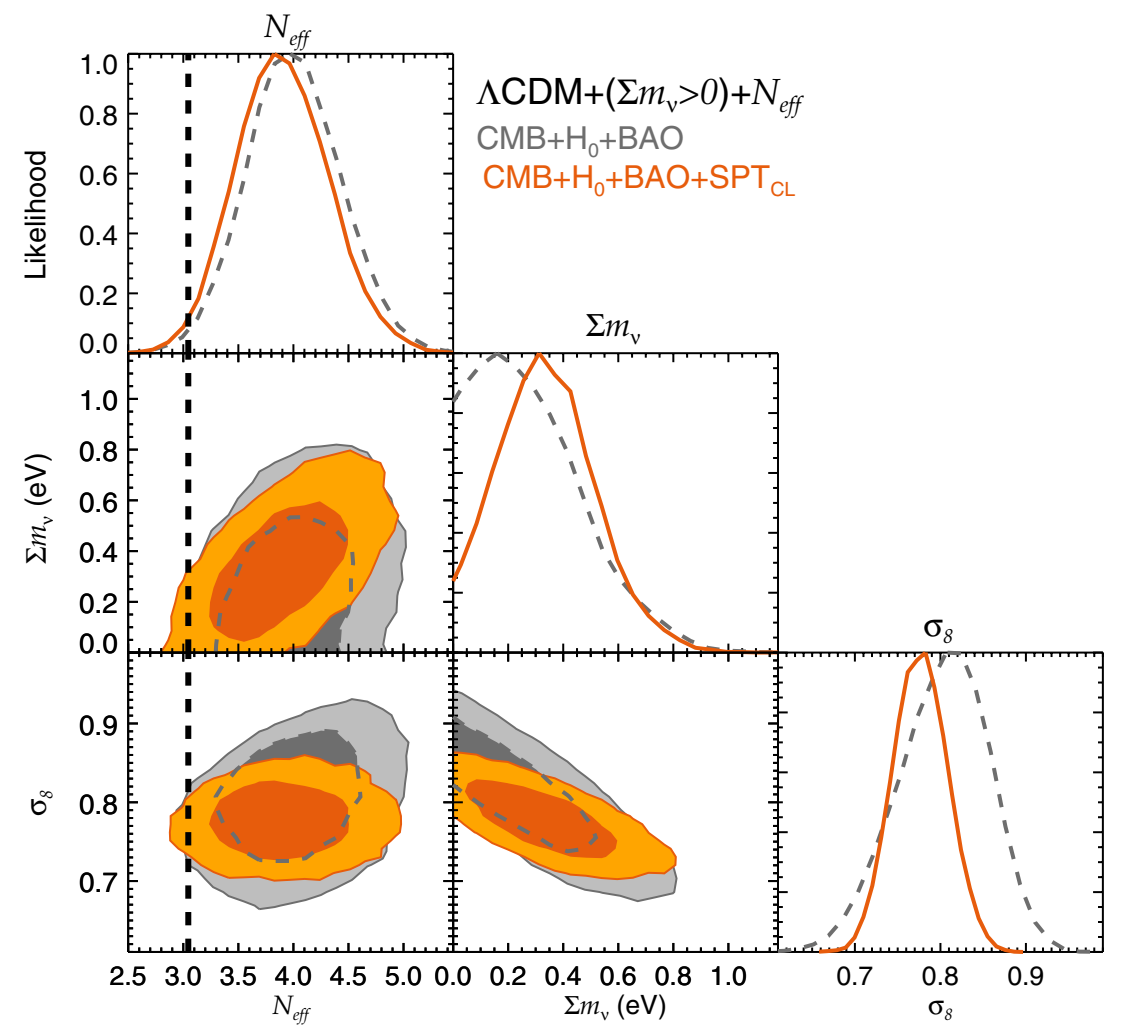

Figure 7. Assuming a $\Lambda \mathrm{CDM}$ cosmology with massive neutrinos and a free number of relativistic species, $N_{\text {eff }}$, the constraints on $\sigma_{8}$, $\Sigma m_{v}$, and $N_{\text {eff }}$. The plots along the diagonal are the one-dimensional marginalized likelihood. The off-diagonal plots are the two-dimensional marginalized constraints showing the $68 \%$ and $95 \%$ confidence regions. We show the constraints for the $\mathrm{CMB}+\mathrm{H}_{0}+\mathrm{BAO}$ (gray, dashed), and $\mathrm{CMB}+\mathrm{H}_{0}+\mathrm{BAO}+\mathrm{SPT} \mathrm{CL}_{\mathrm{CL}}$ (orange, solid) data sets. The black vertical line shows $N_{\text {eff }}=3.046$, the expected value for three neutrino species. Using the $\mathrm{CMB}+H_{0}+\mathrm{BAO}+\mathrm{SPT} \mathrm{CL}$ data set, the $68 \%$ confidence marginalized constraints are $\sigma_{8}=0.777 \pm 0.031, N_{\text {eff }}=3.91 \pm 0.42$, and $\Sigma m_{v}=0.34 \pm 0.17 \mathrm{eV}$, with a 95\% CL of $\Sigma m_{v}<0.63 \mathrm{eV}$.

(A color version of this figure is available in the online journal.)

suppressing structure formation, and lowering $\sigma_{8}$ at $z=0$. Therefore, increasing $N_{\text {eff }}$ will also allow an increasing $\Sigma m_{v}$. Keisler et al. (2011) used a combination of $\mathrm{CMB}+\mathrm{H}_{0}+\mathrm{BAO}$ data to constrain $\Sigma m_{v}<0.69 \mathrm{eV}$ at a $95 \% \mathrm{CL}, \sigma_{8}=0.803 \pm 0.056$, and $N_{\text {eff }}=3.98 \pm 0.43$.

In Figure 7, we show the constraints on $N_{\text {eff }}, \Sigma m_{v}$, and $\sigma_{8}$, using the $\mathrm{CMB}+\mathrm{H}_{0}+\mathrm{BAO}$ data set, before and after including the $\mathrm{SPT}_{\mathrm{CL}}$ data. In Table 5 we give the marginalized constraints. When varying $N_{\text {eff }}$ we assume consistency with BBN for our constraints. Using the $\mathrm{CMB}+\mathrm{H}_{0}+\mathrm{BAO}+\mathrm{SPT}_{\mathrm{CL}}$ data set, we constrain $\Sigma m_{v}<0.63 \mathrm{eV}$ at a 95\% CL, $\sigma_{8}=0.777 \pm 0.031$, and $N_{\text {eff }}=3.91 \pm 0.42$. Relative to Keisler et al. (2011), the addition of the $\mathrm{SPT}_{\mathrm{CL}}$ data improves the constraints on $\sigma_{8}$ by a factor of 1.8, and reduces the upper limit on $\Sigma m_{v}$ by a factor of 1.1. However, the addition of the $\mathrm{SPT}_{\mathrm{CL}}$ data does noticeably sharpen the peak in the marginalized one-dimensional likelihood for $\Sigma m_{v}$, such that the maximum likelihood constraint peaks away from zero, $\Sigma m_{v}=0.34 \pm 0.17 \mathrm{eV}$.

As noted in Keisler et al. (2011), models of the CMB power spectrum that include increased damping are favored at the $1.6 \sigma-1.9 \sigma$ level. However, even if one accepts the need for an extra parameter to explain the damping, its physical origin is unclear. Regardless, considering the $N_{\text {eff }}$ model extension is instructive to help understand the model dependency of the neutrino mass constraints. Keisler et al. (2011) considered three models to explain the excess damping and found that the $N_{\text {eff }}$ model had the most significant effect on $\sigma_{8}$. The inclusion of $N_{\text {eff }}$ also weakens the constraints on $\Sigma m_{\nu}$, because of the degeneracies between $N_{\text {eff }}, \sigma_{8}$, and $\Sigma m_{\nu}$. In the combined cosmological data set, the $\mathrm{SPT}_{\mathrm{CL}}$ data mainly constrain $\sigma_{8}$, which helps to break this degeneracy and indirectly improve the neutrino mass constraints. Therefore, the $\Sigma m_{v}$ constraint from the $N_{\text {eff }}$ model can be considered a conservative upper limit on $\Sigma m_{v}$ regardless of the physical mechanism for the increased damping.

\section{3. $\Lambda C D M$ with $f_{N L}$}

Finally, we consider a $\Lambda \mathrm{CDM}$ cosmology with primordial non-Gaussianity. Standard inflationary cosmology predicts that density fluctuations in the universe were seeded by random Gaussian fluctuations. However, inflationary models can be constructed that predict significant levels of non-Gaussianity (e.g., Bartolo et al. 2004). The leading order non-Gaussian term is typically described by the parameter $f_{\mathrm{NL}}$. Using a measurement of the $\mathrm{CMB}$ power spectrum from WMAP7 data, Komatsu et al. (2011) measured a 95\% CL of $-10<$ $f_{\mathrm{NL}}<74$. Primordial non-Gaussianity can also manifest itself through the abundance of massive galaxy clusters. While the constraints from an SPT-like SZ survey are not expected to be competitive with current CMB constraints (Dalal et al. 2008), they constitute a constraint independent from the CMB results which is sensitive to very different physical scales. In principle, even a single massive high-redshift cluster can falsify standard $\Lambda \mathrm{CDM}$ cosmology (Mortonson et al. 2011), though currently the most massive cluster known at $z>1$ is not in significant tension (Foley et al. 2011).

In our analysis, we incorporate $f_{\mathrm{NL}}$ as a modification of the cluster mass function following the prescription of Dalal et al. 


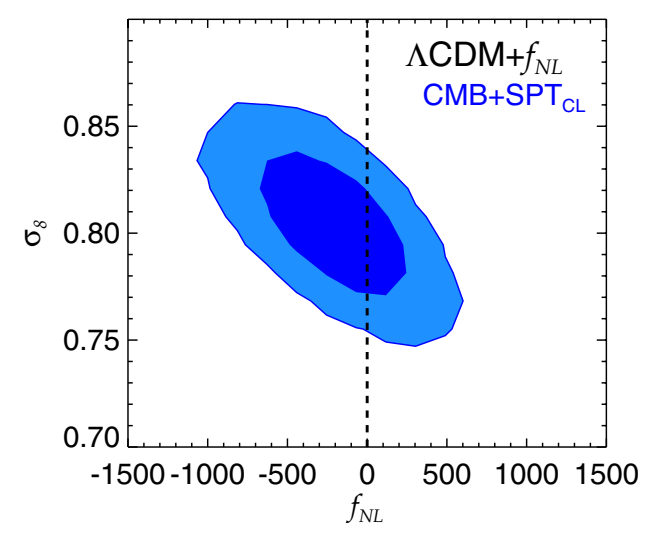

Figure 8. Assuming a $\Lambda \mathrm{CDM}$ cosmology with a primordial non-Gaussianity characterized by the parameter $f_{\mathrm{NL}}$, the two-dimensional marginalized constraints on $\sigma_{8}$ and $f_{\mathrm{NL}}$ using the $\mathrm{CMB}+\mathrm{SPT} \mathrm{CL}_{\mathrm{L}}$ data set (blue). Contours show the $68 \%$ and $95 \%$ confidence regions. We only consider the effect of $f_{\mathrm{NL}}$ on the $\mathrm{SPT}_{\mathrm{CL}}$ data set. We measure $f_{\mathrm{NL}}=-220 \pm 317$, consistent with zero non-Gaussianity.

(A color version of this figure is available in the online journal.)

(2008), and only consider the effect of $f_{\mathrm{NL}}$ on the SPT $\mathrm{CL}_{\mathrm{CL}}$ data set. Using $N$-body simulations, Dalal et al. (2008) calculate a simple fitting function defined by $f_{\mathrm{NL}}$ that modifies the cluster mass function, where cluster masses were calculated from a friendsof-friends (FOF) algorithm with a linking length of $b=0.2$. We modify the fitting function to be appropriate for the cluster masses used in this work by characterizing the relationship between $M_{500}$ and FOF cluster masses, $M_{\text {FOF }}$.

To do this, we use the simulations described in Section 3.1.4, the large-volume dark matter simulations used to characterize the $\zeta-M_{500}$ relation. For each halo, we calculate both $M_{500}$ and $M_{\mathrm{FOF}}$, where we assume the same $b=0.2$ linking length as Dalal et al. (2008). For clusters between $0.3<z<1.2$ and with $M_{500}>1 \times 10^{14} M_{\odot} h^{-1}$, which spans the range of clusters used in this work, we find that on average $M_{500}=0.59 \times M_{\mathrm{FOF}}$, varying by $\lesssim 10 \%$ over this redshift and mass range, and with a standard deviation between halos of $12 \%$. Using this relationship for the average offset and standard deviation, we modify the fitting function of Dalal et al. (2008) to be appropriate for $M_{500}$ cluster masses.

In Figure 8, we show the constraints on $\sigma_{8}$ and $f_{\mathrm{NL}}$ using the $\mathrm{CMB}+\mathrm{SPT}_{\mathrm{CL}}$ data set. The marginalized constraints are $f_{\mathrm{NL}}=$ $-220 \pm 317, \sigma_{8}=0.805 \pm 0.022$, and $A_{\mathrm{SZ}}=5.28 \pm 0.91$. The best-fit value of $f_{\mathrm{NL}}$ is slightly negative, generally implying fewer massive high-redshift clusters. In Section 4, we found that for a $\Lambda C D M$ cosmology using the $\mathrm{CMB}_{+} \mathrm{SPT}_{\mathrm{CL}}$ data, our constraint for $A_{\mathrm{SZ}}$ was $0.9 \sigma$ lower than the simulation prior. This corresponds to a higher mass for a cluster of a given $\xi$, which can also be thought of as the SPT survey having fewer clusters than expected given the simulation prior. When $f_{\mathrm{NL}}$ is added as a parameter, $A_{\mathrm{SZ}}$ moves back toward its simulation prior, and the deficit of clusters can be maintained by a more negative $f_{\mathrm{NL}}$. For the model to match the number of clusters measured by SPT, a more negative $f_{\mathrm{NL}}$ can be balanced with either a larger $\sigma_{8}$ or $A_{\mathrm{SZ}}$, creating a degeneracy in this direction. Regardless, any deficit of clusters is not significant relative to the uncertainty on either $\sigma_{8}$ or $A_{\mathrm{Sz}}$, even in a $\Lambda \mathrm{CDM}$ cosmology for which they are best constrained.

Our results are consistent with Williamson et al. (2011) which used the 26 most massive clusters in the full $2500 \mathrm{deg}^{2}$ SPT-SZ survey to constrain $f_{\mathrm{NL}}=20 \pm 450$. Our work differs from Williamson et al. (2011) in that we use a much smaller area of the SPT-SZ survey, we select clusters down to a lower $\xi$ threshold, and we use an improved mass calibration.

Our constraints on $f_{\mathrm{NL}}$ are negligibly affected by uncertainties in the conversion from $M_{\mathrm{FOF}}$ to $M_{500}$, both because of the fairly weak mass dependence of the fitting function in Dalal et al. (2008) and our relatively poor constraints on $f_{\mathrm{NL}}$. For example, the offset in the fitting function, between the Gaussian and non-Gaussian halo mass, varies as $\sim \sqrt{M}$. Therefore a factorof-two uncertainty in the conversion from $M_{\mathrm{FOF}}$ to $M_{500}$ only causes a $\sim \sqrt{2}$ change in the offset of the fitting function, and a similar $\sim \sqrt{2}$ change in $f_{\mathrm{NL}}$. We have explicitly tested this relationship by running additional MCMC chains with a factor of two different conversion between $M_{\mathrm{FOF}}$ to $M_{500}$, and find that this relationship approximately holds. This level of uncertainty is still negligible to our current constraints on $f_{\mathrm{NL}}$ from the $\mathrm{SPT}_{\mathrm{CL}}$ data set. For similar reasons, the $f_{\mathrm{NL}}$ constraints are fairly robust to uncertainties in cosmology, given the effective priors from the CMB data set, and the resulting uncertainties in the concentration-mass relation.

\section{SOURCES OF UNCERTAINTY}

Previous SPT cluster survey results, namely V10 and Williamson et al. (2011), found cosmological constraints that were limited most significantly by the cluster mass calibration, or equivalently the fractional uncertainty in $A_{\mathrm{SZ}}$. In this work, we have reduced this uncertainty by incorporating the external mass calibration from the $Y_{X}-M_{500}$ relation using X-ray observations of the SPT clusters. We can directly estimate the impact of the uncertainties in the X-ray and SZ scaling relations by importance sampling the MCMC chains, where we post-process the chains by imposing a narrow prior on each scaling relation parameter centered around the best-fit value. The resulting increase in precision on the cosmological parameters allows a measure of the impact from the uncertainty in the scaling relations. In this way, we effectively "fix" the X-ray and scaling relation parameters, a process which we will implicitly be referring to throughout this section. For a $w \mathrm{CDM}$ cosmology, we also consider the impact of the SNe systematic uncertainty on the cosmological results presented here.

With enough SZ and X-ray observations, we expect the $\zeta-M_{500}$ calibration to be limited by the calibration of the $Y_{X}-M_{500}$ relation because the latter has tighter external priors. In practice, there will be an additional uncertainty in the $\zeta-M_{500}$ calibration from the limited number of SZ and $\mathrm{X}$-ray observations for cross-calibration, and this uncertainty will also degrade the cosmological constraints. We wish to separate this effect, which we will refer to as the $\mathrm{SZ}-Y_{X}$ scaling uncertainty, from the additional systematic uncertainty from the $Y_{X}-M_{500}$ calibration, which we will refer to as the $X$-ray scaling uncertainty, and the statistical uncertainty from the cluster sample size. By fixing the X-ray and SZ scaling relation parameters, as described above, we can measure the impact of the SZ- $Y_{X}$ scaling uncertainty, X-ray scaling uncertainty, and statistical uncertainty on our cosmological constraints.

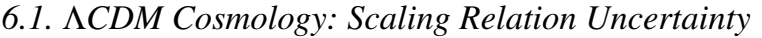

We first consider the $\Lambda \mathrm{CDM}$ constraints using the $\mathrm{SPT}_{\mathrm{CL}}+H_{0}+\mathrm{BBN}$ data set, the results of which were described in Section 4. These data best constrained the combination of $\sigma_{8}\left(\Omega_{m} / 0.25\right)^{0.30}=0.785 \pm 0.037$. The sources of uncertainty for this result are summarized in Table 6 and are discussed below. 
Table 6

Error Budget

\begin{tabular}{|c|c|c|c|c|c|}
\hline & \multirow{2}{*}{$\begin{array}{c}\Lambda \mathrm{CDM} \\
\mathrm{SPT}_{\mathrm{CL}}+H_{0}+\mathrm{BBN} \\
\sigma_{8}\left(\Omega_{m} / 0.25\right)^{0.30}\end{array}$} & \multicolumn{2}{|c|}{$\begin{array}{c}w \mathrm{CDM} \\
\mathrm{SPT}_{\mathrm{CL}}+H_{0}+\mathrm{BBN} \\
\end{array}$} & \multicolumn{2}{|c|}{$\begin{array}{c}w \mathrm{CDM} \\
\mathrm{CMB}+\mathrm{BAO}+\mathrm{SNe}+\mathrm{SPT}_{\mathrm{CL}}\end{array}$} \\
\hline & & $w$ & $\sigma_{8}$ & $w$ & $\sigma_{8}$ \\
\hline Baseline, Sections 4, 5.1 & $0.785 \pm 0.037$ & $-1.09 \pm 0.36$ & $0.773 \pm 0.088$ & $-0.973 \pm 0.063$ & $0.793 \pm 0.028$ \\
\hline SNe systematic & $\ldots$ & & $\ldots$ & \pm 0.026 & \pm 0.005 \\
\hline SZ- $Y_{X}$ scaling & \pm 0.010 & \pm 0.19 & \pm 0.066 & \pm 0.013 & \pm 0.013 \\
\hline $\mathrm{X}$-ray scaling systematic & \pm 0.028 & \pm 0.15 & \pm 0.036 & \pm 0.019 & \pm 0.014 \\
\hline Statistical & \pm 0.023 & \pm 0.27 & \pm 0.046 & \pm 0.033 & \pm 0.013 \\
\hline
\end{tabular}

Notes. We give the mean and 68\% confidence intervals for a subset of the cosmological parameters reported in Sections 4 and 5.1. The last four rows give the $1 \sigma$ error in each cosmological parameter due to the stated uncertainty.

For the X-ray scaling relation parameters, only the uncertainty in $A_{X}$ and $C_{X}$, the normalization and redshift evolution parameters contribute significantly to the uncertainty on $\sigma_{8}\left(\Omega_{m} / 0.25\right)^{0.30}$. Fixing each parameter separately implies that they contribute an uncertainty on $\sigma_{8}\left(\Omega_{m} / 0.25\right)^{0.30}$ of \pm 0.022 and \pm 0.015 , respectively. It is not surprising that the normalization of the mass calibration significantly affects the constraints, and the redshift evolution can be understood for similar reasons. For a cluster at the median redshift of the SPT sample, $z=0.74$, the prior on the $C_{X}$ value effectively contributes an additional $8 \%$ to the cluster mass calibration. This can be compared to the $10 \%$ mass calibration uncertainty from the prior on $A_{X}$. Fixing all X-ray parameters simultaneously, implies that they contribute an uncertainty on $\sigma_{8}\left(\Omega_{m} / 0.25\right)^{0.30}$ of \pm 0.028 .

For the SZ scaling relation parameters, only the uncertainty in $A_{\mathrm{SZ}}$ contributes significantly to the uncertainty on $\sigma_{8}\left(\Omega_{m} / 0.25\right)^{0.30}$. Fixing all the SZ scaling parameters, we measure an uncertainty on $\sigma_{8}\left(\Omega_{m} / 0.25\right)^{0.30}$ of \pm 0.023 from statistical uncertainty, \pm 0.010 from the SZ $-Y_{X}$ scaling uncertainty, and \pm 0.028 due to $X$-ray scaling uncertainty (as discussed above). The relatively low contribution from the SZ $-Y_{X}$ scaling uncertainty is not surprising considering the constraints on the fractional uncertainty of $A_{\mathrm{SZ}}$, which was near the systematic limit of $14 \%$ imposed by the $Y_{X}-M_{500}$ calibration.

Therefore, the $\Lambda \mathrm{CDM}$ constraints are nearly at the systematic limit from the calibration of the $Y_{X}-M_{500}$ relation. For our constraint of $\sigma_{8}\left(\Omega_{m} / 0.25\right)^{0.30}=0.785 \pm 0.037$, the X-ray scaling and statistical uncertainty contribute almost equal amounts of \pm 0.028 and \pm 0.023 , respectively. By only increasing the cluster sample size we could reduce the uncertainty by up to $\sim 1.3$ $(\sim 0.037 / 0.028)$. Further improvements would require a more accurate cluster mass calibration.

In Section 3.1.2, we discussed the calibration of the $Y_{X}-M_{500}$ relation relative to several recent weak-lensing results. The biggest outlier was Applegate et al. (2012), which favored a larger mass calibration by a factor of $1.17 \pm 0.16$. While this is not a statistically significant outlier, we take this offset as a more conservative estimate of the statistical uncertainty on the mass calibration, increasing the uncertainty of our prior on $A_{X}$ by a factor of 1.7 , so that we can directly calculate its effect on our cosmological constraints. Doing this, we find that the $\mathrm{SPT}_{\mathrm{CL}}+H_{0}+\mathrm{BBN}$ constraints are well approximated as $\sigma_{8}\left(\Omega_{m} / 0.25\right)^{0.30}=0.779 \pm 0.047$, a factor of 1.3 increase in uncertainty relative to the constraints assuming our standard priors. This is in good agreement with our estimate that the standard uncertainty in $A_{X}$ is contributing a uncertainty of \pm 0.022 to the constraint.

\section{2. wCDM Cosmology: Scaling Relation and SNe Uncertainty}

We next consider the sources of uncertainty for the $w \mathrm{CDM}$ cosmology discussed in Section 5.1. We will concentrate on using the $\mathrm{SPT}_{\mathrm{CL}}+H_{0}+\mathrm{BBN}$ and $\mathrm{CMB}+\mathrm{BAO}+\mathrm{SNe}+\mathrm{SPT}_{\mathrm{CL}}$ data sets, which produce constraints of $w=-1.09 \pm 0.36$ and $w=-0.973 \pm 0.063$, respectively. The sources of uncertainty for this result are summarized in Table 6 and are discussed below.

We first consider the $\mathrm{SPT}_{\mathrm{CL}}+H_{0}+\mathrm{BBN}$ data set. For the $\mathrm{X}$-ray scaling relation parameters, we again find that the uncertainty in $A_{X}$ and $C_{X}$ contributes the largest uncertainty on $w$. Fixing each parameter independently implies they contribute an uncertainty of $\delta w= \pm 0.10$ and \pm 0.11 , respectively, and a total uncertainty of \pm 0.15 . For the SZ scaling relation parameters, only the uncertainty in $A_{\mathrm{SZ}}$ contributes significantly to the uncertainty on $w$. Fixing all the SZ scaling parameters, we measure an uncertainty on $w$ of \pm 0.27 from statistical uncertainty, \pm 0.19 from the SZ $-Y_{X}$ scaling uncertainty, and \pm 0.15 due to $\mathrm{X}$-ray scaling uncertainty. Therefore, unlike the $\Lambda \mathrm{CDM}$ case, we find that our constraints on $w$ would be significantly improved by adding more clusters and additional $Y_{X}$ measurements. A similar conclusion is reached repeating the above analysis for $\sigma_{8}$. In principle, adding more clusters and $Y_{X}$ measurements would reduce the uncertainty on $w$ and $\sigma_{8}$ to values limited by the $\mathrm{X}$-ray scaling uncertainty. In this limit we should measure $w$ and $\sigma_{8}$ with an uncertainty of $\delta w= \pm 0.15$ and $\delta \sigma_{8}= \pm 0.036$, or $\sim 2.5$ times better than our current constraints.

Following the discussion of the weak-lensing calibration of the $Y_{X}-M_{500}$ relation in Section 6.1, we can directly consider how our results would change with a more uncertain mass calibration by increasing the uncertainty in the normalization of the $Y_{X}-M_{500}$ relation, $A_{X}$, by a factor of 1.7 , motivated by the biggest outlier in the weak-lensing results, Applegate et al. (2012). Doing this, we find that the $\mathrm{SPT}_{\mathrm{CL}}+\mathrm{H}_{0}+\mathrm{BBN}$ data constrain $w=-1.15 \pm 0.43$, a factor of 1.2 increase in uncertainty relative to the constraints assuming our standard priors.

When considering the $\mathrm{CMB}+\mathrm{BAO}+\mathrm{SNe}+\mathrm{SPT}_{\mathrm{CL}}$ data set, we reach qualitatively similar conclusions, however the total uncertainty is significantly lower because of the parameter degeneracies that are broken from the additional data sets. We first re-run the MCMC chains without SNe systematic uncertainty. Fixing all the SZ scaling parameters, we measure a statistical uncertainty of $\delta w= \pm 0.033$, a factor of two improvement relative to the constraints including all systematic uncertainties. Comparing this uncertainty to that with no fixed parameters, we estimate an uncertainty on $w$ of \pm 0.019 from X-ray scaling uncertainty 
and \pm 0.013 from $S Z-Y_{X}$ scaling uncertainty. The addition of the $\mathrm{SPT}_{\mathrm{CL}}$ data also significantly reduces the systematic uncertainty from SNe. Running a CMB+BAO+SNe MCMC chain with and without $\mathrm{SNe}$ systematics, we measure $w=-1.014 \pm 0.078$ and $w=-1.017 \pm 0.050$, respectively. This implies that SNe systematics are contributing an uncertainty of $\delta w= \pm 0.060$. After adding the $\mathrm{SPT}_{\mathrm{CL}}$ data the uncertainty from $\mathrm{SNe}$ systematics is reduced to $\delta w= \pm 0.026$, a factor of $\sim 2.3$ improvement.

Following the previous discussion, we can consider directly how our results would change with a more uncertain mass calibration. If we increased the uncertainty in the normalization of the $Y_{X}-M_{500}$ relation, $A_{X}$, by a factor of 1.7 , motivated by the biggest outlier in the weak-lensing results, Applegate et al. (2012), we constrain $w=-0.979 \pm 0.066$ using the $\mathrm{CMB}+\mathrm{BAO}+\mathrm{SNe}+\mathrm{SPT}_{\mathrm{CL}}$ data set. This is a factor of $1.05 \mathrm{in}-$ crease in the uncertainty in $w$ relative to the constraints assuming our standard priors, indicating that our mass calibration uncertainty is indeed a sub-dominant contribution to the uncertainty in $w$.

\section{3. $\Lambda C D M$ with Massive Neutrinos: Dependence on Cluster Mass Calibration}

The constraints on $\Sigma m_{v}$ are weakly dependent on the assumed $\mathrm{SPT}_{\mathrm{CL}}$ mass calibration uncertainty. Following the discussion in Section 6.1, we consider how our constraints on $\Sigma m_{v}$ would change with a more uncertain mass calibration by increasing the uncertainty in the normalization of the $Y_{X}-M_{500}$ relation, $A_{X}$, by a factor of 1.7 , motivated by the biggest outlier in the weak-lensing results, Applegate et al. (2012). For the results in Section 5.2, assuming a $\Lambda$ CDM cosmology with massive neutrinos, we constrain $\Sigma m_{v}<0.30 \mathrm{eV}$ at $95 \%$ confidence using the $\mathrm{CMB}+\mathrm{H}_{0}+\mathrm{SPT}_{\mathrm{CL}}$ data set, a $7 \%$ increase in uncertainty relative to the constraints assuming our standard priors. If, as in Section 5.2.1, we add $N_{\text {eff }}$ as an additional free parameter, we constrain $\Sigma m_{v}<0.65$ at $95 \%$ confidence using the $\mathrm{CMB}+\mathrm{H}_{0}+\mathrm{BAO}+\mathrm{SPT}_{\mathrm{CL}}$ data set, a $5 \%$ increase relative to the constraints assuming our standard priors.

\subsection{Point Source Contamination}

In V10, it was argued that point source contamination contributed a negligible level of uncertainty relative to the statistical precision of the cluster sample. Since we are using the same cluster sample, we expect the same conclusion to hold, though we summarize their arguments here.

From Poisson distributed sources, the probability of a chance superposition of a bright point source ( $\geq 6 \mathrm{mJy}$ ) with a cluster is negligible, given the sky density of sources at $150 \mathrm{GHz}$ $\left(\sim 1 \mathrm{deg}^{-2}\right.$; Vieira et al. 2010). Furthermore, our cosmological analysis in Section 3 explicitly accounts for a Poisson distributed background of sources. The amount of point source power in the SPT data has been characterized in several CMB power spectrum results (Lueker et al. 2010; Shirokoff et al. 2011; Reichardt et al. 2012a). This power is explicitly accounted for in the construction of the spatial filter used to find clusters in the SPT data. Poisson distributed point sources typically contribute $\sim 0.2 \sigma$ of the noise $(\sim 0.4 \mathrm{mJy})$ in the SPT spatially filtered $150 \mathrm{GHz}$ maps, with the level weakly dependent on the spatial scale of the clusters, effectively acting as another source of noise in the data. In addition, the gravitational lensing of this background by clusters is expected to only marginally increase the noise associated with these sources (Lima et al. 2009), and will be therefore small compared to the intrinsic scatter in the $\zeta-M_{500}$ relation and instrumental noise in the SPT maps.
Point sources that were correlated with the cluster would not be accounted for in the above; however, it is expected that this contribution is negligible. Correlated radio emission has previously been calculated, based on radio observations of nonSZ discovered cluster samples, to be negligible at $150 \mathrm{GHz}$ for clusters of the typical SPT mass scale and redshift range (Lin et al. 2009; Sehgal et al. 2010).

Correlated dusty emission is similarly not expected to be significant through several lines of evidence. We have used IRAS measurements of massive, low-redshift $(z \sim 0.2)$, $\mathrm{X}$-ray-selected clusters and measured the average infrared source flux. When this flux is extrapolated, using well-motivated star-forming galaxy spectral energy distributions (SEDs), to $150 \mathrm{GHz}$, the contamination per cluster is negligible (i.e., $<1$ mJy at $95 \%$ confidence), even with the most pessimistic SED template choices. At higher redshift, Bai et al. (2007) measured that the abundance of infrared-luminous star-forming galaxies in a massive $\left(\gtrsim 10^{15} M_{\odot}\right)$ cluster at $z=0.8$ to be over an order of magnitude lower relative to the field abundance than a simple mass scaling would predict. Combined with sub-millimeter luminosity measurements from BLAST (Pascale et al. 2009), these measurements predict that a sphere with a $1 \mathrm{Mpc}$ radius at $z=1$ with cluster-like infrared emission would produce $<0.1$ mJy of emission at $150 \mathrm{GHz}$.

In addition, using Spitzer observations of X-ray-selected groups and low-mass clusters at $0.5<z<1.0$, George et al. (2011) found that correlated dusty emission is insignificant compared to the SZ signal. Because star formation is expected to be more strongly quenched in more massive cluster environments (Hashimoto et al. 1998), we would expect this conclusion to be even stronger at the higher-mass scales corresponding to SPT clusters. Finally, in McDonald et al. (2012), we describe observations of one SPT cluster at $z=0.596$, SPT-CL J23444243 , that appears to be undergoing a cooling-flow induced burst of star formation in its brightest cluster galaxy (BCG) of $\sim 740 M_{\odot} \mathrm{yr}^{-1}$, over an order of magnitude larger than other low-redshift cool-core clusters. Even in this most pessimistic case for correlated dusty emission, the predicted flux at $150 \mathrm{GHz}$ ( $2 \mathrm{~mm}$ ) is $0.1 \mathrm{mJy}$ from Herschel detections of the BCG at 250, 350 , and $500 \mu \mathrm{m}$. Therefore, through many lines of evidence, it is expected that correlated dusty emission is $\lesssim 0.1 \mathrm{mJy}$, an order of magnitude less than the noise in the SPT maps.

Finally, even though we expect correlated mm-emission from clusters to be negligible, as argued above, the addition of the $\mathrm{X}$-ray measurements in the cosmological MCMC analysis in Section 3 allows this bias to be corrected for. An offset in the true normalization of the $\zeta-M_{500}$ relation from the central value of its prior, due to point sources or otherwise, will be adjusted for in the MCMC analysis, because of the relatively large uncertainty on the normalization of the $\zeta-M_{500}$ relation (30\%) compared to the $Y_{X}-M_{500}$ relation $(10 \%)$.

\section{DISCUSSION}

\subsection{Improvement Relative to V10}

In this work, the cluster sample is the same as used in V10 for their cosmological analysis. However, we have improved the cosmological constraints relative to V10 by including X-ray measurements in order to reduce the cluster mass calibration uncertainty. It is not straightforward to quantify the improvement for two main reasons. First, V10 used pre-existing WMAP7 MCMC chains from Komatsu et al. (2011) that they importance sampled by re-weighting the chains by the likelihood of 
the SPT cluster catalog given each set of parameters. In this work, we generated new MCMC chains while simultaneously fitting both data sets. Second, each result uses somewhat different external data sets, in particular for the CMB power spectrum measurements, where the results in this work also include CMB measurements from Keisler et al. (2011).

Without explicitly correcting for these differences, we can approximate the improvement from including the X-ray measurements by considering the relative improvements of adding the $\mathrm{SPT}_{\mathrm{CL}}$ data to the CMB data used in either result. For a $\Lambda \mathrm{CDM}$ cosmology, the WMAP7 data constrain $\sigma_{8}=0.801 \pm 0.030$. In V10, the addition of the SPT data, this constraint improved to $\sigma_{8}=0.791 \pm 0.027$, a factor of 1.1 improvement. In this work, for a $\Lambda$ CDM cosmology using the $\mathrm{SPT}_{\mathrm{CL}}$ and CMB data, we constrained $\sigma_{8}=0.795 \pm 0.016$, a factor of 1.5 improvement over the constraints from the CMB alone. Therefore, the addition of the X-ray measurements improved the $\Lambda \mathrm{CDM}$ constraints on $\sigma_{8}$ by a factor of $\sim 1.4$. A comparison of the $w \mathrm{CDM}$ cosmological constraints is more complicated because of the somewhat different handling of the external data sets. Regardless, the significant improvement in the constraints from the $\mathrm{X}$-ray measurements is clear.

\subsection{Prospects for Further Improvement}

The results in this paper were derived using 18 clusters from $178 \mathrm{deg}^{2}$ of the $2500 \mathrm{deg}^{2}$ SPT-SZ survey. The full survey will significantly increase both the area and overall depth of the SZ maps. Reichardt et al. (2012b) have presented a catalog 158 confirmed clusters from the first $720 \mathrm{deg}^{2}$ of the SPT-SZ survey, with a median redshift of $\sim 0.55$ and a median mass of $M_{500} \sim 2.3 \times 10^{14} M_{\odot} h^{-1}$. This sample is representative of the cluster yield and properties for the full survey, which was completed in 2011 November and has detected $\sim 500$ clusters. Therefore it is useful to consider how the method used in this work will be applied to the full survey, and what level of improvement we can expect on the cosmological constraints.

Using the $\mathrm{SPT}_{\mathrm{CL}}+H_{0}+\mathrm{BBN}$ data set, we found that our constraints are currently limited by both statistical uncertainty and the SZ $-Y_{X}$ scaling uncertainty. Both uncertainties would be improved by adding more SPT clusters with additional $Y_{X}$ measurements. Recently the SPT collaboration was awarded a Chandra X-ray Visionary Project (XVP) to complete X-ray observations of the 80 most significant clusters at $z>0.4$ detected in the first $2000 \mathrm{deg}^{2}$ of the SPT-SZ survey. As argued in Section 5.1.2, we would need $\gtrsim 50$ clusters with $Y_{X}$ measurements for the $\zeta-M_{500}$ calibration to be limited by the $Y_{X}-M_{500}$ uncertainty. With these many clusters, the statistical uncertainty on $w$ should decrease to a level below the systematic uncertainty from the X-ray scaling relation, $\delta w= \pm 0.15$. Combining the full $2500 \mathrm{deg}^{2}$ SPT-SZ survey with the Chandra XVP observations, we would be limited to this constraint from the current calibration of the $Y_{X}-M_{500}$ relation.

To reduce the systematic uncertainty further, we would need more accurate cluster mass estimates than currently exist from $\mathrm{X}$-ray measurements alone. In Section 6.2, we found that the $\mathrm{X}$-ray scaling systematics were currently limited by the uncertainty in $A_{X}$ and $C_{X}$, whose fractional uncertainty was $10 \%$ and $50 \%$, respectively. Reducing their uncertainty by a factor of two would reduce their contribution to the systematic uncertainty to $\delta w= \pm 0.037$ and \pm 0.074 , respectively. This would effectively correspond to an overall mass calibration uncertainty of $5 \%$ with an additional $6 \%$ uncertainty in the evolution of the mass calibration between $z=0.0-1.1$.
This level of mass calibration should be achievable by incorporating additional data sets, such as optical velocity dispersion (White et al. 2010) or weak-lensing measurements (Hoekstra 2007; Becker \& Kravtsov 2011). For example, in massive clusters, the scatter in weak-lensing mass estimates is expected to be $\sim 20 \%$ (Becker \& Kravtsov 2011). Therefore, with weak-lensing observations of two sets of $\sim 15-20$ clusters at low and high redshift, this level of accuracy should be achievable. Toward this goal, the SPT collaboration has been approved for weak-lensing observations of $\sim 35$ SPT-detected clusters spanning $0.30<z<1.3$ using the Magellan and Hubble telescopes. Additionally, the SPT collaboration has been approved for optical velocity dispersion observations of $\sim 100$ SPT-detected clusters using the Very Large Telescope (VLT) and a large NOAO program on Gemini South. With these data sets, we expect to achieve the factor of two improvement in mass calibration, as discussed above.

Applying this calibration to the full $2500 \mathrm{deg}^{2} \mathrm{SPT}_{\mathrm{CL}}+\mathrm{H}_{0}+$ BBN data set, we should constrain $w$ with an accuracy of $\sim 8 \%$, or a factor of $\sim 4.5$ tighter than the current $\mathrm{SPT}_{\mathrm{CL}}+\mathrm{H}_{0}+\mathrm{BBN}$ constraints. This improved constraint would be comparable to the current constraints from the $\mathrm{CMB}+\mathrm{BAO}+\mathrm{SNe}$ data, and would be an independent systematic test of the standard dark energy paradigm by measuring the effect of dark energy on the growth of structure. Combining the existing $\mathrm{CMB}+\mathrm{BAO}+\mathrm{SNe}$ data with the $2500 \mathrm{deg}^{2}$ SPT cluster sample, the uncertainty from the SZ $-Y_{X}$ scaling and the cluster sample size is expected to be negligible compared to the uncertainty contributed by the improved cluster mass calibration. In this case, the SPT cluster data would contribute an uncertainty of only $\sim 1 \%$ to the significantly improved constraint on $w$ from the combined data set.

\section{CONCLUSIONS}

We use measurements from the SPT-SZ cluster survey in combination with X-ray measurements to constrain cosmological parameters. We have described and implemented a method that simultaneously fits for cosmological parameters and the scaling of the SZ and X-ray observables with cluster mass. The method is generalizable to multiple cluster observables, and self-consistently accounts for the effects of cluster selection and uncertainties in cluster mass calibration on the derived cosmological constraints. We apply this method to an SZ-selected catalog of 18 galaxy clusters identified in $178 \mathrm{deg}^{2}$ of the $2500 \mathrm{deg}^{2}$ SPT-SZ survey. This is the first analysis of an SZ survey to directly incorporate X-ray observations, which has reduced the uncertainty on both the cluster mass calibration and the cosmological constraints.

For a $\Lambda \mathrm{CDM}$ cosmology, we find that the $\mathrm{SPT}_{\mathrm{CL}}+\mathrm{H}_{0}+\mathrm{BBN}$ data best constrain $\sigma_{8}\left(\Omega_{m} / 0.25\right)^{0.30}=0.785 \pm 0.037$, where the total uncertainty consists of an approximately equal amount of statistical and systematic uncertainty. These constraints are consistent, and comparable, with other constraints using X-ray-selected (Vikhlinin et al. 2009b; Mantz et al. 2010c) and optically-selected (Rozo et al. 2010) cluster samples. In combination with measurements of the CMB power spectrum from the SPT data and the seven-year WMAP data, the SPT cluster data constrain $\sigma_{8}=0.795 \pm 0.016$ and $\Omega_{m}=0.255 \pm$ 0.016 , a factor of 1.5 improvement on each parameter over the constraints from the CMB data alone.

We consider several extensions beyond a $\Lambda$ CDM cosmological model by including the following as free parameters: the dark energy equation of state $(w)$, the sum of the neutrino masses 
$\left(\Sigma m_{v}\right)$, the effective number of relativistic species $\left(N_{\text {eff }}\right)$, and a primordial non-Gaussianity $\left(f_{\mathrm{NL}}\right)$.

For a $w \mathrm{CDM}$ cosmology, the $\mathrm{SPT}_{\mathrm{CL}}+H_{0}+\mathrm{BBN}$ data constrain $w=-1.09 \pm 0.36$ and $\sigma_{8}=0.773 \pm 0.088$, consistent with dark energy being due to a cosmological constant, and with comparable uncertainties to constraints from the $\mathrm{CMB}$ data alone. Using the $\mathrm{CMB}+\mathrm{BAO}+\mathrm{SNe}+\mathrm{SPT}_{\mathrm{CL}}$ data set, we constrain $w=-0.973 \pm 0.063$ and $\sigma_{8}=0.793 \pm 0.028$, a factor of 1.25 and 1.4 improvement, respectively, over the constraints without SPT cluster data. The uncertainty on $w$ consists of approximately equal contributions from statistical uncertainty, systematic uncertainty from $\mathrm{SNe}$, and systematic uncertainty from cluster scaling relations, with the latter contributing an uncertainty of $\delta w= \pm 0.023$.

We next consider a $\Lambda \mathrm{CDM}$ cosmology with a non-zero neutrino mass. Using a $\mathrm{CMB}+\mathrm{H}_{0}+\mathrm{BAO}+\mathrm{SPT}_{\mathrm{CL}}$ data set, we constrain the sum of the neutrino masses $\Sigma m_{v}$ to be $<0.33 \mathrm{eV}$ at $95 \%$ confidence, a factor of 1.4 improvement over the constraints without SPT cluster data. We find even tighter constraints when we exclude the BAO data set, which tend to favor a lower value of $H_{0}$ and therefore a higher neutrino mass. Using a $\mathrm{CMB}+H_{0}+\mathrm{SPT}_{\mathrm{CL}}$ data set, we constrain $\Sigma m_{v}<$ $0.28 \mathrm{eV}$ at $95 \%$ confidence. We also consider a model with a free effective number of relativistic species, $N_{\text {eff }}$, to explain the increased damping that is observed in the $\mathrm{CMB}$ power spectrum. Using a $\mathrm{CMB}+\mathrm{H}_{0}+\mathrm{BAO}+\mathrm{SPT}_{\mathrm{CL}}$ data set, we jointly measure $N_{\text {eff }}=3.91 \pm 0.42$ and $\Sigma m_{v}=0.34 \pm 0.17 \mathrm{eV}$, while constraining $\Sigma m_{v}<0.63 \mathrm{eV}$ at $95 \%$ confidence.

Finally, we consider a $\Lambda \mathrm{CDM}$ cosmology where we allow the number of observed clusters to be affected by non-Gaussian density fluctuations characterized by the parameter $f_{\mathrm{NL}}$. Using a $\mathrm{CMB}_{+} \mathrm{SPT}_{\mathrm{CL}}$ data set, we measure $f_{\mathrm{NL}}=-220 \pm 317$, consistent with zero non-Gaussianity.

The results presented in this paper use 18 clusters from $178 \mathrm{deg}^{2}$ of the $2500 \mathrm{deg}^{2}$ SPT-SZ survey, and are limited by the combination of the cluster sample size and mass calibration. The SPT-SZ survey was completed in 2011 November, and has detected $\sim 500$ clusters with a median redshift of $\sim 0.5$ and a median mass of $M_{500} \sim 2.3 \times 10^{14} M_{\odot} h^{-1}$. Ongoing $\mathrm{X}$-ray, weak-lensing, and optical velocity dispersion observations of SPT-SZ-selected clusters will be used to produce an improved cluster mass calibration of the sample. The full SPT-SZ survey and improved mass calibration will produce constraints on $w$ comparable to current constraints from the combination of $\mathrm{CMB}+\mathrm{BAO}+\mathrm{SNe}$ data, and would represent an independent systematic test of the standard dark energy paradigm by measuring the effect of dark energy on the growth of structure. The combination of $\mathrm{CMB}+\mathrm{BAO}+\mathrm{SNe}$ data with the SPT cluster sample will break degeneracies between the data sets, resulting in significantly tighter constraints on dark energy.

The South Pole Telescope program is supported by the National Science Foundation through grant ANT-0638937. Partial support is also provided by the NSF Physics Frontier Center grant PHY-0114422 to the Kavli Institute of Cosmological Physics at the University of Chicago, the Kavli Foundation, and the Gordon and Betty Moore Foundation. Additional data were obtained with the $6.5 \mathrm{~m}$ Magellan Telescopes located at the Las Campanas Observatory, Chile. Support for X-ray analysis was provided by NASA through Chandra Award Numbers 12800071, 12800088, and 13800883 issued by the Chandra X-ray Observatory Center, which is operated by the Smithsonian Astrophysical Observatory for and on behalf of NASA
Table 7

X-Ray Observables for SPT Clusters

\begin{tabular}{lcrccc}
\hline \hline Name & $z$ & \multicolumn{1}{c}{$\begin{array}{r}r_{500} \\
(\mathrm{kpc})\end{array}$} & $\begin{array}{c}T_{X} \\
(\mathrm{keV})\end{array}$ & $\begin{array}{c}M_{g} \\
\left(10^{13} M_{\odot}\right)\end{array}$ & $\begin{array}{c}Y_{X} \\
\left(10^{14} M_{\odot} \mathrm{keV}\right)\end{array}$ \\
\hline SPT-CL J0509-5342 $^{\mathrm{a}}$ & 0.463 & $1062 \pm 39$ & $7.0_{-1.1}^{+1.4}$ & $5.6_{-0.2}^{+0.2}$ & $4.3 \pm 0.8$ \\
SPT-CL J0528-5300 $^{\mathrm{b}}$ & 0.765 & $765 \pm 47$ & $5.2_{-1.2}^{+1.9}$ & $2.8_{-0.3}^{+0.3}$ & $1.6 \pm 0.5$ \\
SPT-CL J0533-5005 $^{\mathrm{b}}$ & 0.881 & $666 \pm 51$ & $3.9_{-1.1}^{+1.6}$ & $2.3_{-0.4}^{+0.5}$ & $1.0 \pm 0.4$ \\
SPT-CL J0546-5345 $^{\mathrm{b}}$ & 1.067 & $823 \pm 27$ & $6.8_{-0.9}^{+1.2}$ & $7.4_{-0.3}^{+0.4}$ & $4.8 \pm 0.8$ \\
SPT-CL J0551-5709 $^{\mathrm{b}}$ & 0.423 & $923 \pm 34$ & $4.0_{-0.6}^{+0.6}$ & $5.1_{-0.6}^{+0.6}$ & $1.9 \pm 0.4$ \\
SPT-CL J0559-5249 $^{\mathrm{a}}$ & 0.611 & $1071 \pm 30$ & $7.7_{-0.8}^{+1.1}$ & $8.3_{-0.2}^{+0.3}$ & $6.4 \pm 0.8$ \\
SPT-CL J2331-5051 $^{\mathrm{a}}$ & 0.571 & $972 \pm 34$ & $5.9_{-0.8}^{+1.3}$ & $5.7_{-0.2}^{+0.2}$ & $3.5 \pm 0.6$ \\
SPT-CL J2332-5358 $^{\mathrm{c}}$ & 0.403 & $1166 \pm 31$ & $7.8_{-0.9}^{+1.0}$ & $7.6_{-0.3}^{+0.2}$ & $6.1 \pm 0.8$ \\
SPT-CL J2337-5942 $^{\mathrm{a}}$ & 0.781 & $1046 \pm 39$ & $8.9_{-1.4}^{+2.0}$ & $9.5_{-0.6}^{+0.4}$ & $8.5 \pm 1.7$ \\
SPT-CL J2341-5119 $^{\mathrm{a}}$ & 0.998 & $847 \pm 37$ & $8.0_{-1.6}^{+1.9}$ & $5.6_{-0.2}^{+0.2}$ & $4.7 \pm 1.0$ \\
SPT-CL J2342-5411 $^{\mathrm{c}}$ & 1.074 & $648 \pm 29$ & $5.0_{-0.8}^{+0.9}$ & $2.6_{-0.3}^{+0.3}$ & $1.4 \pm 0.3$ \\
SPT-CL J2355-5056 & 0.320 & $997 \pm 31$ & $5.3_{-0.7}^{+0.9}$ & $3.9_{-0.1}^{+0.2}$ & $2.2 \pm 0.4$ \\
SPT-CL J2359-5009 $^{\mathrm{b}, \mathrm{c}}$ & 0.774 & $778 \pm 36$ & $5.2_{-0.9}^{+1.3}$ & $3.1_{-0.3}^{+0.3}$ & $1.8 \pm 0.4$ \\
SPT-CL J0000-5748 & 0.701 & $950 \pm 68$ & $8.3_{-2.2}^{+3.6}$ & $4.4_{-0.5}^{+0.5}$ & $4.2 \pm 1.6$ \\
\hline
\end{tabular}

Notes. X-ray observables for clusters with Chandra or XMM-Newton observations. For clusters with new spectroscopic redshifts or new Chandra observations, we have recalculated their X-ray observables, as described in Section 2.1.3. To maintain consistency with A11, all X-ray observables are calculated assuming a preferred $\Lambda \mathrm{CDM}$ cosmology using $W M A P 7+\mathrm{BAO}+\mathrm{H}_{0}$ data with $\Omega_{M}=0.272, \Omega_{\Lambda}=0.728$, and $H_{0}=70.2 \mathrm{~km} \mathrm{~s}^{-1} \mathrm{Mpc}^{-1}$ (Komatsu et al. 2011).

a X-ray observables taken from A11.

${ }^{\mathrm{b}}$ Updated for new Chandra observations.

${ }^{c}$ Updated for new spectroscopic redshift.

under contract NAS8-03060. Optical imaging data from the Blanco $4 \mathrm{~m}$ at Cerro Tololo Interamerican Observatories (programs 2005B-0043, 2009B-0400, 2010A-0441, 2010B-0598) and spectroscopic observations from VLT programs 086.A0741 and 286.A-5021 and Gemini program GS-2009B-Q-16 were included in this work. We acknowledge the use of the Legacy Archive for Microwave Background Data Analysis (LAMBDA). Support for LAMBDA is provided by the NASA Office of Space Science. Galaxy cluster research at Harvard is supported by NSF grant AST-1009012. Galaxy cluster research at SAO is supported in part by NSF grants AST-1009649 and MRI-0723073. The McGill group acknowledges funding from the National Sciences and Engineering Research Council of Canada, Canada Research Chairs program, and the Canadian Institute for Advanced Research. X-ray research at the CfA is supported through NASA Contract NAS 8-03060. This work is based in part on observations made with the Spitzer Space Telescope, which is operated by the Jet Propulsion Laboratory, California Institute of Technology under a contract with NASA. Support for this work was provided by NASA through an award issued by JPL/Caltech. The Munich group acknowledges support from the Excellence Cluster Universe and the DFG research program TR33. R.J.F. is supported by a Clay Fellowship. B.A.B. is supported by a KICP Fellowship, M.B. acknowledges support from contract 2834-MIT-SAO-4018 from the Pennsylvania State University to the Massachusetts Institute of Technology. M.D. acknowledges support from an Alfred P. Sloan Research Fellowship, W.F. and C.J. acknowledge support from the Smithsonian Institution, and B.S. acknowledges support from the Brinson Foundation. 
Table 8

Clusters with New Chandra X-Ray Observations

\begin{tabular}{|c|c|c|c|c|c|c|}
\hline \multirow[t]{2}{*}{ Name } & \multirow[t]{2}{*}{$z$} & \multicolumn{2}{|c|}{ A11 } & \multicolumn{2}{|c|}{ This Work } & \multirow[t]{2}{*}{ ObsIDs } \\
\hline & & $\begin{array}{c}\text { Exposure } \\
(\mathrm{ks})\end{array}$ & $\begin{array}{l}\text { Source } \\
\text { Counts }\end{array}$ & $\begin{array}{c}\text { Exposure } \\
(\mathrm{ks})\end{array}$ & $\begin{array}{l}\text { Source } \\
\text { Counts }\end{array}$ & \\
\hline SPT-CL J0528-5300 & 0.765 & 36.5 & 356 & 115.9 & 1732 & $9341,10862,11996, \mathbf{1 1 7 4 7}, \mathbf{1 1 8 7 4}, \mathbf{1 2 0 9 2}, \mathbf{1 3 1 2 6}$ \\
\hline SPT-CL J0533-5005 & 0.881 & 41.5 & 201 & 67.7 & 344 & $11748,12001, \mathbf{1 2 0 0 2}$ \\
\hline SPT-CL J0546-5345 & 1.067 & 55.6 & 1304 & 67.8 & 1512 & 9332, 9336, 10851, 10864, 11739 \\
\hline SPT-CL J0551-5709 & 0.423 & 19.8 & 876 & 33.2 & 1212 & $11871, \mathbf{1 1 7 4 3}$ \\
\hline
\end{tabular}

Notes. The ObsIDs refer to all the Chandra observations used in this work. The ObsIDs that are new, relative to A11, are highlighted in bold. For clusters not listed here, we use the same Chandra and XMM-Newton observations as listed by A11.

Facilities: Blanco (MOSAIC), CXO (ACIS), Gemini:South (GMOS), Magellan:Baade (IMACS), Magellan:Clay (LDSS3), Spitzer (IRAC), SPT, XMM (EPIC)

\section{APPENDIX A}

\section{X-RAY OBSERVATIONS AND RESULTS}

In Table 7, we give updated X-ray observables for the clusters used in this work, as discussed in Section 2.1.3. For the five clusters without new measurements, we give the results from A11 directly, in order to provide a complete listing for the cluster sample. In Table 8, we give the complete list of Chandra observation identifications (ObsIDs) used for clusters with new Chandra observations.

\section{APPENDIX B}

\section{LIKELIHOOD MODIFICATION TO ACCOUNT FOR COSMOLOGICAL DEPENDENCE OF $Y_{X}$}

In Section 3.2 we presented a procedure for translating the theoretical mass function, $d N / d M d z$, into observable space, $d N / d \xi d Y_{X} d z$. Under the assumption that this transformation is independent of the cosmological and scaling relation parameters $\boldsymbol{p}$ we are ultimately trying to recover, this procedure modifies the log likelihood by a constant offset.

However, in the case of $Y_{X}$, this assumption ceases to hold true as $Y_{X}$ is a derived quantity, calculated explicitly for each new value of $\boldsymbol{p}$ (i.e., at each likelihood evaluation in the MCMC). This added subtlety can be addressed in the following fashion. Let us define $Y_{X}^{*}$ as the value of $Y_{X}$ when evaluated at some reference point in parameter space $\boldsymbol{p}^{*}$. In order for the probability contained in a differential volume to be independent of a change of variables, we need to multiply by the Jacobian of the transformation as follows:

$$
\begin{aligned}
P\left(z, \xi, \boldsymbol{Y}_{x}^{*} \mid \boldsymbol{p}\right) & =\left|\frac{\partial\left(\boldsymbol{Y}_{x}\right)}{\partial\left(\boldsymbol{Y}_{x}^{*}\right)}\right| P\left(z, \xi, \boldsymbol{Y}_{x} \mid \boldsymbol{p}\right) \\
& =\left|\prod_{i} f_{i}(\boldsymbol{p})\right| P\left(z, \xi, \boldsymbol{Y}_{x} \mid \boldsymbol{p}\right),
\end{aligned}
$$

where $f_{i}(\boldsymbol{p})$ is the ratio of the $Y_{X}$ value of the $i$ th cluster at $\boldsymbol{p}$ to its value at $\boldsymbol{p}^{*}$. Expressing this in terms of log probability, and ignoring constant offsets, we obtain

$$
\ln P\left(z, \xi, \boldsymbol{Y}_{x} \mid \boldsymbol{p}\right)=\ln P\left(z, \xi, \boldsymbol{Y}_{x}^{*} \mid \boldsymbol{p}\right)+\sum_{i} \ln Y_{x, i} .
$$

Table 9

Mass Estimates for the SPT Cluster Catalog

\begin{tabular}{lrlc}
\hline \hline Object Name & $\xi$ & $z$ & $\begin{array}{c}M_{500}\left(\rho_{\text {crit }}\right) \\
\left(10^{14} M_{\odot} h_{70}^{-1}\right)\end{array}$ \\
\hline SPT-CL J0509-5342 $^{\mathrm{a}}$ & 6.61 & 0.463 & $5.11 \pm 0.68$ \\
SPT-CL J0511-5154 $^{\mathrm{a}}$ & 5.63 & 0.74 & $3.36 \pm 0.86$ \\
SPT-CL J0521-5104 $^{\mathrm{a}}$ & 5.45 & 0.72 & $3.21 \pm 0.86$ \\
SPT-CL J0528-5259 $^{\text {SPT-CL J0533-5005 }}$ & 5.45 & 0.765 & $2.96 \pm 0.54$ \\
SPT-CL J0539-5744 $^{\mathrm{a}}$ & 5.59 & 0.881 & $2.54 \pm 0.54$ \\
SPT-CL J0546-5345 $^{\text {SPT-CL J0551-5709 }}$ & 5.12 & 0.77 & $2.93 \pm 0.86$ \\
SPT-CL J0559-5249 $^{\text {SPT-CL J2301-5546 }}$ & 7.69 & 1.067 & $4.79 \pm 0.64$ \\
SPT-CL J2331-5051 & 6.13 & 0.423 & $3.61 \pm 0.54$ \\
SPT-CL J2332-5358 & 9.28 & 0.611 & $6.36 \pm 0.79$ \\
SPT-CL J2337-5942 & 5.19 & 0.748 & $3.00 \pm 0.86$ \\
SPT-CL J2341-5119 & 8.04 & 0.572 & $4.89 \pm 0.68$ \\
SPT-CL J2342-5411 & 7.30 & 0.403 & $6.21 \pm 0.79$ \\
SPT-CL J2355-5056 & 14.94 & 0.781 & $7.68 \pm 1.04$ \\
SPT-CL J2359-5009 & 9.65 & 0.998 & $5.14 \pm 0.71$ \\
SPT-CL J0000-5748 & 6.18 & 1.074 & $2.75 \pm 0.46$ \\
& 5.89 & 0.320 & $3.96 \pm 0.54$ \\
& 6.35 & 0.774 & $3.32 \pm 0.54$ \\
& 5.48 & 0.701 & $4.04 \pm 0.68$
\end{tabular}

Note. ${ }^{\text {a }}$ These clusters have only SZ data, and no X-ray observations.

This results in the straightforward prescription of adding $\sum \ln Y_{X}$ to the likelihood at each step in the MCMC, a process very similar to that suggested and performed in Mantz et al. (2008) and Vikhlinin et al. (2009a).

\section{APPENDIX C}

\section{MASS ESTIMATES}

We present posterior mass estimates for all 18 clusters considered in this work in Table 9. Where applicable, these are joint X-ray and SZ posterior mass estimates, for clusters without X-ray data we use the SZ posterior mass estimate. We calculate a probability density function on a mass grid at each point in the $\Lambda \mathrm{CDM}$ chain that was calculated using the $\mathrm{CMB}+\mathrm{SPT}_{\mathrm{CL}}$ data, from Section 4.1. The probability density functions are combined to obtain a mass estimate that has been fully marginalized over all cosmological and scaling relation parameters. We report the mean and the $68 \%$ confidence interval for the mass estimate.

\section{REFERENCES}

Aguilar-Arevalo, A. A., Anderson, C. E., Brice, S. J., et al. 2010, PhRvL, 105, 181801

Amanullah, R., Lidman, C., Rubin, D., et al. 2010, ApJ, 716, 712 
Andersson, K., Benson, B. A., Ade, P. A. R., et al. 2011, ApJ, 738, 48 Applegate, D. E., von der Linden, A., Kelly, P. L., et al. 2012, arXiv:1208.0605 Arnaud, M., Pratt, G. W., Piffaretti, R., et al. 2010, A\&A, 517, A92

Bai, L., Marcillac, D., Rieke, G. H., et al. 2007, ApJ, 664, 181 Bartolo, N., Komatsu, E., Matarrese, S., \& Riotto, A. 2004, PhR, 402, 103 Bashinsky, S., \& Seljak, U. 2004, PhRvD, 69, 083002

Becker, M. R., \& Kravtsov, A. V. 2011, ApJ, 740, 25

Bhattacharya, S., Heitmann, K., White, M., et al. 2011, ApJ, 732, 122

Bode, P., Ostriker, J. P., Weller, J., \& Shaw, L. 2007, ApJ, 663, 139

Brodwin, M., Ruel, J., Ade, P. A. R., et al. 2010, ApJ, 721, 90

Carlstrom, J. E., Ade, P. A. R., Aird, K. A., et al. 2011, PASP, 123, 568

Carlstrom, J. E., Holder, G. P., \& Reese, E. D. 2002, ARA\&A, 40, 643

Dalal, N., Doré, O., Huterer, D., \& Shirokov, A. 2008, PhRvD, 77, 123514

Dicus, D. A., Kolb, E. W., Gleeson, A. M., et al. 1982, PhRvD, 26, 2694

Dobbs, M. A., Lueker, M., Aird, K. A., et al. 2012, RScI, 89, 3113

Dunkley, J., Hlozek, R., Sievers, J., et al. 2011, ApJ, 739, 52

Fabjan, D., Borgani, S., Rasia, E., et al. 2011, MNRAS, 416, 801

Foley, R. J., Andersson, K., Bazin, G., et al. 2011, ApJ, 731, 86

George, M. R., Leauthaud, A., Bundy, K., et al. 2011, ApJ, 742, 22

Haehnelt, M. G., \& Tegmark, M. 1996, MNRAS, 279, 545

Haiman, Z., Mohr, J. J., \& Holder, G. P. 2001, ApJ, 553, 545

Hashimoto, Y., Oemler, A., Jr., Lin, H., \& Tucker, D. L. 1998, ApJ, 499, 589

High, F. W., Hoekstra, H., Leethochawalit, N., et al. 2012, ApJ, 758, 22

High, F. W., Stalder, B., Song, J., et al. 2010, ApJ, 723, 1736

Hoekstra, H. 2007, MNRAS, 379, 317

Hoekstra, H., Mahdavi, A., Babul, A., \& Bildfell, C. 2012, MNRAS, 427, 1298

Holder, G., Haiman, Z., \& Mohr, J. J. 2001, ApJL, 560, 111

Hou, Z., Keisler, R., Knox, L., Millea, M., \& Reichardt, C. 2011, arXiv: 1104.2333

Ichikawa, K., Fukugita, M., \& Kawasaki, M. 2005, PhRvD, 71, 043001

Jeltema, T. E., Hallman, E. J., Burns, J. O., \& Motl, P. M. 2008, ApJ, 681, 167

Jenkins, A., Frenk, C. S., White, S. D. M., et al. 2001, MNRAS, 321, 372

Keisler, R., Reichardt, C. L., Aird, K. A., et al. 2011, ApJ, 743, 28

Kelly, P. L., von der Linden, A., Applegate, D. E., et al. 2012, arXiv:1208.0602

Kirkman, D., Tytler, D., Suzuki, N., O'Meara, J. M., \& Lubin, D. 2003, ApJS, 149,1

Komatsu, E., Dunkley, J., Nolta, M. R., et al. 2009, ApJS, 180, 330

Komatsu, E., Smith, K. M., Dunkley, J., et al. 2011, ApJS, 192, 18

Kopp, J., Maltoni, M., \& Schwetz, T. 2011, PhRvL, 107, 091801

Kravtsov, A. V., Vikhlinin, A., \& Nagai, D. 2006, ApJ, 650, 128

Larson, D., Dunkley, J., Hinshaw, G., et al. 2011, ApJS, 192, 16

Lewis, A., \& Bridle, S. 2002, PhRvD, 66, 103511

Lewis, A., Challinor, A., \& Lasenby, A. 2000, ApJ, 538, 473

Lima, M., Jain, B., \& Devlin, M. 2009, MNRAS, 406, 2352

Lin, Y., Partridge, B., Pober, J. C., et al. 2009, ApJ, 694, 992

Linder, E. V., \& Jenkins, A. 2003, MNRAS, 346, 573

Lopez, R. E., Dodelson, S., Heckler, A., \& Turner, M. S. 1999, PhRvL, 82, 3952

Lueker, M., Reichardt, C. L., Schaffer, K. K., et al. 2010, ApJ, 719, 1045

Mahdavi, A., Hoekstra, H., Babul, A., \& Henry, J. P. 2008, MNRAS, 384, 1567

Mahdavi, A., Hoekstra, H., Babul, A., et al. 2012, arXiv:1210.3689

Mangano, G., Miele, G., Pastor, S., et al. 2005, NuPhB, 729, 221
Mantz, A., Allen, S. W., Ebeling, H., \& Rapetti, D. 2008, MNRAS, 387, 1179

Mantz, A., Allen, S. W., Ebeling, H., Rapetti, D., \& Drlica-Wagner, A. 2010a, MNRAS, 406, 1773

Mantz, A., Allen, S. W., \& Rapetti, D. 2010b, MNRAS, 406, 1805

Mantz, A., Allen, S. W., Rapetti, D., \& Ebeling, H. 2010c, MNRAS, 406, 1759

Marriage, T. A., Acquaviva, V., Ade, P. A. R., et al. 2011, ApJ, 737, 61

Marrone, D. P., Smith, G. P., Okabe, N., et al. 2012, ApJ, 754, 119

McDonald, M., Bayliss, M., Benson, B. A., et al. 2012, Natur, 488, 349

Melin, J.-B., Bartlett, J. G., \& Delabrouille, J. 2006, A\&A, 459, 341

Mention, G., Fechner, M., Lasserre, T., et al. 2011, PhRvD, 83, 073006

Mortonson, M. J., Hu, W., \& Huterer, D. 2011, PhRvD, 83, 023015

Nagai, D., Kravtsov, A. V., \& Vikhlinin, A. 2007, ApJ, 668, 1

Nelson, K., Rudd, D. H., Shaw, L., \& Nagai, D. 2012, ApJ, 751, 121

Ngeow, C. C., Mohr, J., Zenteno, A., et al. 2009, BAAS, 41, 335

Okabe, N., Takada, M., Umetsu, K., Futamase, T., \& Smith, G. P. 2010, PASJ, 62, 811

Padin, S., Staniszewski, Z., Keisler, R., et al. 2008, ApOpt, 47, 4418

Pascale, E., Ade, P. A. R., Bock, J. J., et al. 2009, ApJ, 707, 1740

Percival, W. J., Reid, B. A., Eisenstein, D. J., et al. 2010, MNRAS, 401, 2148

Planck Collaboration, Ade, P. A. R., Aghanim, N., Arnaud, M., et al. 2011, A\&A, 536, A8

Poole, G. B., Babul, A., McCarthy, I. G., et al. 2007, MNRAS, 380, 437

Rasia, E., Mazzotta, P., Evrard, A., et al. 2011, ApJ, 729, 45

Reichardt, C. L., Shaw, L., Zahn, O., et al. 2012a, ApJ, 755, 70

Reichardt, C. L., Stalder, B., Bleem, L. E., et al. 2012b, arXiv: 1203.5775

Reid, B. A., Verde, L., Jimenez, R., \& Mena, O. 2010, JCAP, 01, 003

Riess, A. G., Macri, L., Casertano, S., et al. 2011, ApJ, 730, 119

Rozo, E., Wechsler, R. H., Rykoff, E. S., et al. 2010, ApJ, 708, 645

Sehgal, N., Bode, P., Das, S., et al. 2010, ApJ, 709, 920

Sehgal, N., Trac, H., Acquaviva, V., et al. 2011, ApJ, 732, 44

Shaw, L. D., Holder, G. P., \& Bode, P. 2008, ApJ, 686, 206

Shaw, L. D., Zahn, O., Holder, G. P., \& Doré, O. 2009, ApJ, 702, 368

Shirokoff, E., Reichardt, C. L., Shaw, L., et al. 2011, ApJ, 736, 61

Short, C. J., Thomas, P. A., Young, O. E., et al. 2010, MNRAS, 408, 2213

Springel, V. 2005, MNRAS, 364, 1105

Springel, V., White, S. D. M., Jenkins, A., et al. 2005, Natur, 435, 629

Stanek, R., Rasia, E., Evrard, A. E., Pearce, F., \& Gazzola, L. 2010, ApJ, 715 , 1508

Staniszewski, Z., Ade, P. A. R., Aird, K. A., et al. 2009, ApJ, 701, 32

Sunyaev, R. A., \& Zel'dovich, Y. B. 1972, CoASP, 4, 173

Tinker, J., Kravtsov, A. V., Klypin, A., et al. 2008, ApJ, 688, 709

Vanderlinde, K., Crawford, T. M., de Haan, T., et al. 2010, ApJ, 722, 1180

Vieira, J. D., Crawford, T. M., Switzer, E. R., et al. 2010, ApJ, 719, 763

Vikhlinin, A., Burenin, R. A., Ebeling, H., et al. 2009a, ApJ, 692, 1033

Vikhlinin, A., Kravtsov, A. V., Burenin, R. A., et al. 2009b, ApJ, 692, 1060

von der Linden, A., Allen, M. T., Applegate, D. E., et al. 2012, arXiv:1208.0597

Wang, L., \& Steinhardt, P. J. 1998, ApJ, 508, 483

Warren, M. S., Abazajian, K., Holz, D. E., \& Teodoro, L. 2006, ApJ, 646, 881

White, M., Cohn, J. D., \& Smit, R. 2010, MNRAS, 408, 1818

Williamson, R., Benson, B. A., High, F. W., et al. 2011, ApJ, 738, 139

Zhang, Y.-Y., Finoguenov, A., Bohringer, H., et al. 2008, A\&A, 482, 451 